\title{
Multi-Objective Overtaking Maneuver Planning for Autonomous Ground Vehicles
}

\author{
Runqi Chai, Member, IEEE, Antonios Tsourdos, Member, IEEE, Al Savvaris, Senchun Chai, Senior Member, IEEE, Yuanqing \\ Xia, Senior Member, IEEE, and C. L. Philip Chen, Fellow, IEEE,
}

\begin{abstract}
Constrained autonomous vehicle overtaking trajectories are usually difficult to be generated due to certain practical requirements and complex environmental limitations. This problem becomes more challenging when multiple contradicting objectives are required to be optimized and the on-road objects to be overtaken are irregularlyplaced. In this paper, a novel swarm intelligence-based algorithm is proposed for producing the multi-objective optimal overtaking trajectory of autonomous ground vehicles. The proposed method solves a multiobjective optimal control model in order to optimize the maneuver time duration, the trajectory smoothness, and the vehicle visibility, while taking into account different types of mission-dependent constraints. However, one problem that could have an impact on the optimization process is the selection of algorithm control parameters. To desensitize the negative influence, a novel fuzzy adaptive strategy is proposed and embedded in the algorithm framework. This allows the optimization process can dynamically balance the local exploitation and global exploration, thereby exploring the trade-off between objectives more effective. The performance of using the designed fuzzy adaptive multi-objective method is analyzed and validated by executing a number of simulation studies. The results confirm the effectiveness of applying the proposed algorithm to produce multi-objective optimal overtaking trajectories for the autonomous ground vehicles. Moreover, the comparison to other stateof-the-art multi-objective optimization schemes shows that the designed strategy tends to be more capable in terms of producing a set of widespread and high-quality pareto-optimal solutions.
\end{abstract}

Index Terms-Autonomous vehicle, overtaking trajectories, multiobjective, irregularly-placed, swarm intelligence, fuzzy adaptive strategy, pareto-optimal.

\section{INTRODUCTION}

A UTONOMOUS vehicle (AV) motion planning has received great attention over the last decade due to its increasing importance in the design of advanced autonomous control systems. Early studies on this subject were primarily focused on the development and implementation of geometric motion planners [1]-[3]. It has been shown in a large amount of work that it is possible and effective to use these planners to produce a feasible path that can achieve a prespecified target and fulfill the mission [4], [5]. However, one critical drawback of applying the geometric methods is that the consideration of mission constraints is often problematic and the constraint handling way might vary from problem to problem.

To effectively deal with this problem and offer an alternative, in recent years, there has been a growing interest in solving the motion planning problems via the optimization-based technique [6], [7]. One important advantage of using this technique is that it has

R. Chai, A. Tsourdos and A. Savvaris are with the School of Aerospace, Transport and Manufacturing, Cranfield University, UK, e-mail: (r.chai@cranfield.ac.uk), (a.tsourdos@cranfield.ac.uk), and (a.savvaris@cranfield.ac.uk).

S. Chai and Y. Xia are with the school of Automation, Beijing Institute of Technology, Beijing, China, e-mail: (chaisc97@163.com), (xia_yuanqing@bit.edu.cn).

C. L. P. Chen is with the Faculty of Science and Technology, University of Macau, Macau 999078, China, also with the Department of Navigation, Dalian Maritime University, Dalian 116026, China, and also with the State Key Laboratory of Management and Control for Complex Systems, Institute of Automation, Chinese Academy of Sciences, Beijing 100080, China, e-mail: (philip.chen@ieee.org). the capability to model different mission-dependent requirements into constraints, which can then be entailed in the optimization formulation. Contributions made to design or apply optimizationbased motion planners are available in the literature [8]-[11]. For instance, in [8], a space maneuver vehicle regional observation trajectory was designed by applying a desensitized motion optimizer. In [10] the authors applied an interior-point method to evaluate the time-optimal trajectory of an automatic parking problem. Besides, a tractor-trailer motion planning problem was considered in [11], wherein a dynamic programming approach was utilized to optimize the vehicle maneuver profile in a cluttered environment. However, the aforementioned investigations only optimized one single mission objective. In many engineering practices, it is usually demanded by the engineers to include more than one performance index during the optimization phase [12], [13]. Hence, new multi-objective motion planners for autonomous vehicles are highly desired. This will not only benefit the development of autonomous vehicle but also the development of control algorithm in complex ecosystem of smart city.

The problem investigated in this research is a multi-objective optimal overtaking maneuver planning of the autonomous ground vehicle. This type of problem is usually regarded as an extension of classical optimal control problems in the sense that multiple contradicting objectives are taken into account [14]. Overtaking maneuvers are one of the most conventional behaviors in automatic driving. To perform such a maneuver, various physical and environmental limitations are required to be aware. This problem becomes even challenging when the on-road objects to be overtaken are irregularlyplaced. As a result, the difficulty of formulating the optimization model might be increased. Furthermore, due to the complexity of the optimization model, traditional optimization techniques might suffer from convergence problems, thus resulting in premature convergence or infeasible solution detection.

Nature-inspired optimization (NIO) methods are a wide range of various algorithms which are often used to address complex engineering optimization problems that cannot be effectively solved by traditional optimization techniques [15]-[17]. There are a large amount of recently published multi-objective NIO methods which are available in the literature [18]-[20]. In [18], a gradient-based hybrid genetic algorithm was proposed and applied to address an aeroassisted space vehicle trajectory planning problem. In addition, the authors in [19] developed a discrete artificial bee colony algorithm in order to address a multi-objective flexible job-shop scheduling task under the consideration of maintenance activities. In their follow-up research [20], a distributed flow shop scheduling problem was considered and addressed by applying an improved artificial bee colony algorithm.

Among the NIO, particle swarm optimization (PSO) and multiobjective PSO (MOPSO) approaches have been widely researched and applied in the autonomous vehicle motion planning field [21][24]. For example, an unmanned aerial vehicle coordination task was established and studied in [22], wherein a modified simulated binary crossover-based PSO algorithm was proposed to maximize the vehicle controllability. Similarly, in [23], a parallel approach incorporating 
genetic algorithm and PSO was designed so as to produce the shortest path for the autonomous unmanned vehicle in a 3D environment. In addition, an adaptive gradient-based PSO method was formulated in [24]. This algorithm was then applied to generate the time-optimal parking maneuver command for the autonomous ground vehicle. In PSO and MOPSO, each particle among the swarm will be attracted toward its own best position and the global best position. This searching mechanism is likely to result in a higher probability for the algorithm to locate the global optimal solution. Moreover, based on the result presented in [25], the MOPSO has the capability to produce the full pareto solution of all the benchmark problems. Due to these advantages, we give more attention to the implementation of PSO/MOPSO-based techniques.

It should be noted that according to some investigations [25], [26], one potential problem of applying PSO-based techniques is that the evolutionary process tends to be relatively sensitive with respect to the algorithm control parameters. To address this concern, this paper proposes an enhanced MOPSO approach for generating the multi-objective optimal overtaking trajectory of the autonomous ground vehicle. One unique feature of the proposed method is that a novel fuzzy adaptive law is applied to adjust the algorithm control parameters such that the local exploitation and global exploration of the optimization process can be dynamically balanced.

To the best of the authors' knowledge, the present study is a first attempt to develop an enhanced MOPSO for addressing the highly constrained, nonlinear, and high-dimensional autonomous vehicle overtaking maneuver planning problem. Also, this paper contains the following three main contributions:

1) A new multi-objective autonomous vehicle overtaking maneuver optimization model is established.

2) A novel fuzzy adaptive law to adjust the control parameters of the MOPSO algorithm is designed.

3) Extensive case studies and comparative analysis are provided to demonstrate the effectiveness and superiority of the proposed method.

The rest of this paper is organized as follows. The multiobjective optimization formulation used to represent the autonomous vehicle overtaking maneuver planning problem is constructed in Section II. In Section III, a detailed illustration of the proposed fuzzy adaptive MOPSO algorithm is presented. Section IV displays the obtained results including the pareto-optimal solutions and the best compromised solution. The concluding remark is given in Section V.

\section{Multi-ObJective Optimization Formulation of the PROBLEM}

In this section, the mission scenario studied in this work is outlined. Generally speaking, the core aim of the considered problem is to determine a feasible trajectory, for a given autonomous vehicle, to overtake irregularly-placed on-road objects while optimizing multiple pre-defined mission objectives. During the maneuver planning process, several physical limitations are required to be taken into account. These limitations will be modeled into constraints and adopted to find the optimal movement. Hence, one important step is to formulate the optimization problem used throughout this research. Motivated by previous work reported in the literature [10], [11], [24], a novel multi-objective overtaking maneuver planning model is established in this paper. This will be detailed in the following subsections.

\section{A. Vehicle Equations of Motion}

A graphical illustration of the overtaking scenario can be found in Fig.1, from where it is obvious that the entire overtaking maneuver

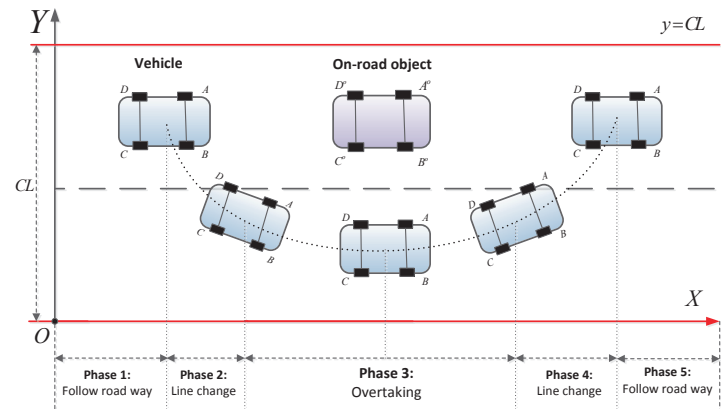

Fig. 1: Graphical illustration of the overtaking scenario.

can be divided into five phases: initial follow roadway, first lane change, overtaking, second lane change, and final follow roadway. To describe the movement of the autonomous vehicle, its equations of motion are given by the following system of differential equations [24]:

$$
\left\{\begin{array}{l}
\frac{d p_{x}}{d t}=v(t) \cos (\theta(t)) \\
\frac{d p_{y}}{d t}=v(t) \sin (\theta(t)) \\
\frac{d v}{d t}=a(t) \\
\frac{d a}{d t}=\eta(t) \\
\frac{d \theta}{d t}=v(t) \tan (\phi(t)) / l \\
\frac{d \phi}{d t}=\omega(t)
\end{array}\right.
$$

where $t \in\left[0, t_{f}\right] .\left[p_{x}, p_{y}, v, a, \theta, \phi\right]$ is the state vector of the vehicle, denoting the central position of the rear wheel, velocity, acceleration, oriental angle and steering angle, respectively. $u=[\eta, \omega]$ stands for the control input, consisting of the jerk variable and the angular velocity of the front wheel.

\section{B. Multiple Objectives}

In this paper, to optimally plan the overtaking maneuver, three mission objectives are taken into consideration. The first objective $J_{1}$ to be minimized is the overtaking time duration such that the vehicle can fulfill the task in the shortest possible time. The other important goal $J_{2}$ is to plan a motion for the autonomous vehicle such that the visibility ahead of the on-road object can be optimized. Fig. 2 provides an illustration regarding the visible and blind areas of the vehicle when an object is irregularly-placed on the road. Some definitions of the vehicle/obstacle-related parameters appeared in Fig. 2 are tabulated in Table I.

TABLE I: Notations for parameters

\begin{tabular}{ll}
\hline \hline$l:$ & Length between the read and front wheels \\
$m:$ & Rear overhang \\
$n:$ & Front overhang \\
$C L:$ & Width of the road \\
$2 b:$ & Width of the vehicle \\
$\psi_{o b}:$ & Observation angle \\
$x_{A}^{o}, y_{A}^{o}$ & Position of $A^{o}$ \\
\hline
\end{tabular}

As can be seen from Fig.2, maximizing the field-of-view is equivalent to maximizing the observation angle $\psi_{o b}(t)$ during the maneuver. Furthermore, a path smoothness indicator $J_{3}$ is proposed and minimized. The aim for minimizing this indicator is to improve the comfort of the passengers and driver. Hence, three objective functions applied for experiments are:

$$
\begin{aligned}
& J_{1}=\min \int_{0}^{t_{f}} d t \\
& J_{2}=\min \int_{0}^{t_{f}}-\psi_{o b}(t) d t \\
& J_{3}=\min \int_{0}^{t_{f}} \omega /\left(l \cos ^{2}(\theta)\right) d t
\end{aligned}
$$




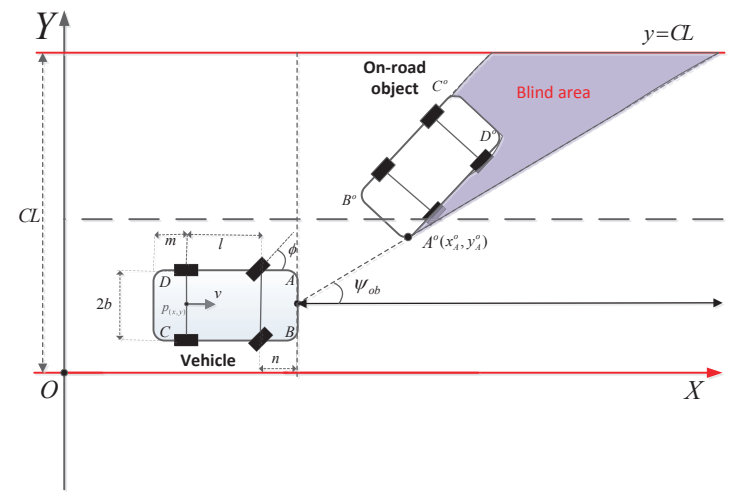

Fig. 2: Illustration of the vehicle and obstacle.

\section{Mission Constraints}

1) State/Control Variable Path Constraints: To take safety requirements and physical limitations of the vehicle into account, a number of state/control variable path constraints should be satisfied, which is given by:

$$
\begin{array}{rr}
p_{x}(t) \in\left[p_{x}^{\text {min }}, p_{x}^{\text {max }}\right] & p_{y}(t) \in\left[p_{y}^{\text {min }}, p_{y}^{\max }\right] \\
v(t) \in\left[v^{\text {min }}, v^{\text {max }}\right] & a(t) \in\left[a^{\text {min }}, a^{\text {max }}\right] \\
\theta(t) \in\left[\theta^{\text {min }}, \theta^{\text {max }}\right] & \phi(t) \in\left[\phi^{\text {min }}, \phi^{\text {max }}\right] \\
\eta(t) \in\left[d_{a}^{\text {min }}, d_{a}^{\text {max }}\right] & \dot{k}(t) \in\left[d_{k}^{\text {min }}, d_{k}^{\text {max }}\right]
\end{array}
$$

where $k=\tan (\theta) / l$ and $\dot{k}=\omega /\left(l \cos ^{2}(\theta)\right)$ are, respectively, the instantaneous curvature and its derivative value. To limit and smoother the acceleration profile, path constraints on the acceleration variable $a$ and jerk variable $\eta$ are imposed. It is worth noting that in [27], Mohseni provided the definition of comfort for autonomous vehicle. Non-comfort was described by high acceleration and jerk values. Hence, we keep using these two criteria to guarantee the comfort to passengers. Moreover, as shown in (3), a path constraint on $\dot{k}$ is also imposed in this paper. The aim is to remove non-smooth parts on the overtaking maneuver profile, thereby improving comfort to passengers further.

2) Road Boundary Constraints: According to Fig.2, the vehicle is considered as a rectangular in the 2-D plane and the four corner points $(\mathrm{ABCD})$ can be calculated via:

$$
\left\{\begin{array}{l}
A_{x}(t)=p_{x}(t)+\cos (\theta(t))(l+n)-b \sin (\theta(t)) \\
A_{y}(t)=p_{y}(t)+\sin (\theta(t))(l+n)+b \cos (\theta(t)) \\
B_{x}(t)=p_{x}(t)+\cos (\theta(t))(l+n)+b \sin (\theta(t)) \\
B_{y}(t)=p_{y}(t)+\sin (\theta(t))(l+n)-b \cos (\theta(t)) \\
C_{x}(t)=p_{x}(t)-m \cos (\theta(t))+b \sin (\theta(t)) \\
C_{y}(t)=p_{y}(t)-m \sin (\theta(t))-b \cos (\theta(t)) \\
D_{x}(t)=p_{x}(t)-m \cos (\theta(t))-b \sin (\theta(t)) \\
\left.D_{y}(t)=p_{y}(t)-m \sin (\theta(t))\right)+b \cos (\theta(t))
\end{array}\right.
$$

During the overtaking maneuver, the autonomous vehicle should not move outside the edge of the road. This is achieved by formulating the following road boundary constraint:

$$
\left\{\begin{array}{l}
0 \leq A_{y}(t) \leq C_{L} \\
0 \leq B_{y}(t) \leq C_{L} \\
0 \leq C_{y}(t) \leq C_{L} \\
0 \leq D_{y}(t) \leq C_{L}
\end{array}\right.
$$

3) Collision-free Constraints: In order to achieve the overtaking maneuver without colliding with other on-road objects, collision-free constraints should be imposed. This paper applies a corner pointbased obstacle avoidance modelling method as illustrated in Fig.3(b) [24]. That is, the corner points of the obstacle $\left(A^{o}, B^{o}, C^{o}, D^{o}\right)$ are located outside $A B C D$ (e.g., the rectangular area of $\mathrm{AV}$ ) during the maneuver. To achieve this, the following inequality is used:

$$
\begin{aligned}
& S_{A A^{\circ} B}+S_{B A^{\circ} C}+S_{C A^{\circ} D}+S_{A A^{\circ} D}>S_{A B C D} \\
& S_{A B^{\circ} B}+S_{B B^{\circ} C}+S_{C B^{\circ} D}+S_{A B^{\circ} D}>S_{A B C D} \\
& S_{A C^{\circ} B}+S_{B C^{\circ} C}+S_{C C^{\circ} D}+S_{A C^{\circ} D}>S_{A B C D} \\
& S_{A D^{\circ} B}+S_{B D^{\circ} C}+S_{C D^{\circ} D}+S_{A D^{\circ} D}>S_{A B C D}
\end{aligned}
$$

where $S_{(\cdot)}$ stands for the area operation.

Remark 1. It is worth noting that in [14], a multi-objective space shuttle reentry mission was considered and a distance-based obstacle avoidance modelling method was applied to deal with the collision avoidance constraint. The general idea of this strategy is to restrict the distance between the center of the vehicle and the obstacle. This method can also be applied in this paper. Specifically, as indicated in Fig.3(a), if the the distance between point $O$ and $O^{o}$ is greater than $r_{v}+r_{o}$ (e.g., Dis $\geq r_{v}+r_{o}$ ), then the vehicle will not collide with the obstacle. The geometric center of the vehicle $\left(O_{x}, O_{y}\right)$ can be calculated by $O_{x}=\left(p_{x}+(((n-m+l) \cos \theta) / 2)\right)$, and $O_{y}=$ $\left(p_{y}+(((n-m+l) \sin \theta) / 2)\right)$. Compared with the corner pointbased strategy, the distance-based method results in less number of constraints. However, as shown in Fig.3, the conservatism of this method might be higher than that of the corner point-based method.

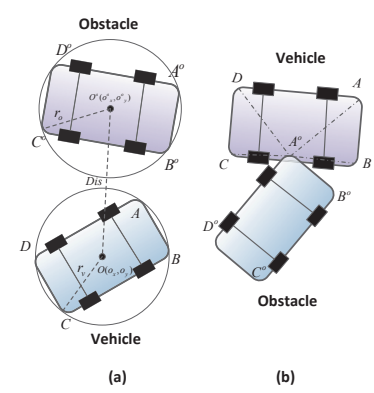

Fig. 3: Collision-free constraint handling methods.

4) Terminal Constraints: The vehicle triggers the maneuver phase at an initial driving condition $x_{0}=\left[p_{x}(0), p_{y}(0), v(0), a(0), \theta(0), \phi(0)\right]$. Once the overtaking phase is completed, it is desired for the vehicle to terminate the entire maneuver at a point where the normal driving condition can be satisfied. Thus, the state terminal boundary constraints are assigned as:

$$
\left[p_{y}\left(t_{f}\right), v\left(t_{f}\right), a\left(t_{f}\right), \theta\left(t_{f}\right)\right]=\left[p_{y}(0), v(0), a(0), \theta(0)\right]
$$

\section{Overall Optimization Formulation}

Based on the construction of equations of motion, multiple objectives and various mission constraints, the multi-objective overtaking maneuver optimization formulation is given by:

$$
\begin{aligned}
& \text { minimize } \quad J=\left[J_{1}, J_{2}, J_{3}\right] \\
& \text { subject to } \forall t \in\left[0, t_{f}\right] \\
& \frac{d p_{x}}{d t}=v(t) \cos (\theta(t)) \\
& \frac{d p y}{d t}=v(t) \sin (\theta(t)) \\
& \frac{d v}{d t}=a(t) \\
& \frac{d a}{d t}=\eta(t) \\
& \frac{d \theta}{d t}=v(t) \tan (\phi(t)) / l \\
& \frac{d t}{d t}=\omega(t) \\
& 0 \leq A_{y}(t) \leq C_{L}, 0 \leq B_{y}(t) \leq C_{L} \\
& 0 \leq C_{y}(t) \leq C_{L}, 0 \leq D_{y}(t) \leq C_{L} \\
& S_{A A^{\circ} B}+S_{B A^{\circ} C}+S_{C A^{\circ} D}+S_{A A^{\circ} D}>S_{A B C D} \\
& S_{A B^{\circ} B}+S_{B B^{\circ} C}+S_{C B^{\circ} D}+S_{A B^{\circ} D}>S_{A B C D} \\
& S_{A C^{\circ} B}+S_{B C^{o} C}+S_{C C^{o} D}+S_{A C^{o} D}>S_{A B C D} \\
& S_{A D^{\circ} B}+S_{B D^{\circ} C}+S_{C D^{\circ} D}+S_{A D^{o} D}>S_{A B C D} \\
& {\left[p_{y}\left(t_{f}\right), v\left(t_{f}\right), a\left(t_{f}\right), \theta\left(t_{f}\right)\right]=} \\
& {\left[p_{y}(0), v(0), a(0), \theta(0)\right]} \\
& p_{x}(t) \in\left[p_{x}^{\text {min }}, p_{x}^{\text {max }}\right], p_{y}(t) \in\left[p_{y}^{\text {min }}, p_{y}^{\max }\right] \\
& v(t) \in\left[v^{\text {min }}, v^{\text {max }}\right], a(t) \in\left[a^{\text {min }}, a^{\text {max }}\right] \\
& \theta(t) \in\left[\theta^{\text {min }}, \theta^{\text {max }}\right], \phi(t) \in\left[\phi^{\text {min }}, \phi^{\text {max }}\right] \\
& \eta(t) \in\left[d_{a}^{\text {min }}, d_{a}^{\max }\right], \dot{k}(t) \in\left[d_{k}^{\text {min }}, d_{k}^{\max }\right]
\end{aligned}
$$




\section{Fuzzy Adaptive Multi-Objective Particle Swarm Optimization ALgORITHM}

In this section, an optimization-based maneuver planning approach is presented to solve the problem defined by (8). This approach, named fuzzy adaptive multi-objective particle swarm optimization (FAMOPSO), can be treated as an enhanced version of the PSO-based motion planner reported in [24]. One unique feature of the proposed approach is that a novel fuzzy adaptive strategy is designed in order to achieve a trade-off between local exploitation and global exploration. Before to introduce the FAMOPSO method in detail, some background information of the constrained MOPSO will be recalled for completeness reasons in the following two subsections.

\section{A. Basic MOPSO Structure}

PSO is one of the nature-inspired optimization methods, which was shown in [24] to be effective for solving complex autonomous vehicle maneuver planning problems. Among the swarm, each particle is treated as a candidate solution. The particle consists of a position vector $u$ and a velocity vector $v$, which can be written as:

$$
\left\{\begin{array}{l}
q_{j}(s)=\left[q_{j, 1}(s), q_{j, 2}(s) \ldots, q_{j, D}(s)\right] \\
v_{j}(s)=\left[v_{j, 1}(s), v_{j, 2}(s) \ldots, v_{j, D}(s)\right]
\end{array}\right.
$$

in which $s=1,2, \ldots, S^{\max }$ represents the number of generation, whereas $D$ is the dimensionally index of the solution space. $j=$ $1,2, \ldots, N_{j}$ stands for the index of the particle. Here, $N_{j}$ is the size of the swarm.

During the evolution, $g(s)=\left[g_{1}(s), \ldots, g_{D}(s)\right]$ and $p_{j}(s)=$ $\left[p_{j, 1}(s), p_{j, 2}(s), \ldots, p_{j, D}(s)\right]$ represent, respectively, the best position in the $s$ th generation and the best position of the $j$ th particle. Applying the information of $g(s)$ and $p_{j}(s)$, the velocity vector of the $j$ th particle is updated via:

$$
\begin{aligned}
v_{j, d}(s+1)= & \omega v_{j, d}(s)+r_{1} c_{1}\left(p_{j, d}(s)-q_{j, d}(s)\right) \\
& r_{2} c_{2}\left(g_{d}(s)-q_{j, d}(s)\right)
\end{aligned}
$$

In (10), $\omega$ denotes the inertia weight, whereas $r_{1}, r_{2}$ are two random positive constants. $d \in\{1,2, \ldots, D\} ;\left[c_{1}, c_{2}\right]$ is the acceleration parameter. Specifically, $c_{1}$ corresponds to the cognitive component, while $c_{2}$ corresponds to the social component. Based on (10), the updated position vector of the $j$ th particle is then defined by:

$$
q_{j, d}(s+1)=q_{j, d}(s)+v_{j, d}(s+1)
$$

It should be noted that in a multi-objective optimization task, we are interested in finding a set of solutions in the sense of pareto-optimal (non-dominant solutions) [14]. As a result, one main difference between the single-objective PSO and MOPSO is that the best position $p_{j}(s)$ is re-recorded based on the domination relationship $\prec$, which could be written as:

$$
p_{j}(s)=\left\{\begin{array}{lll}
q_{j}(s) & \text { if } \quad q_{j}(s) \prec p_{j}(s-1) \\
p_{j}(s-1) & \text { if } \quad q_{j}(s) \nprec p_{j}(s-1)
\end{array}\right.
$$

In (12), $q_{j}(s) \prec p_{j}(s-1)$ means $q_{j}(s)$ is dominated by $p_{j}(s-1)$ and it should be replaced. Defining $A(s)=$ $\left[a_{1}(s), a_{2}(s), \ldots, a_{N_{A}}(s)\right]$ as the external archive, to update the archive $A(s)$, the previous archive $A(s-1)$ and $p_{j}(s)$ are required. That is, for any $j, A(s)=A(s-1) \cup p_{j}(s)$ if $q_{j}(s-1) \prec \succ p_{j}(s)$. Otherwise, the elements that are dominated by $p_{j}(s)$ are firstly removed from $A(s-1)$. Then, $A(s-1)$ is augmented by $p_{j}(s)$. This evolutionary procedure will continue until the termination condition is triggered (e.g., the maximum allowable iteration number is reached $\left.s=S^{\max }\right)$.

\section{B. Constraint Handling}

As many engineering optimization tasks may require to consider different mission constraints, one critical step for the MOPSO algorithm is to deal with the particle infeasibility. For the overtaking maneuver optimization problem, we apply a V-based constraint handling method suggested in [14]. The motivation for the use of V-based constraint handling strategy relies on its ability in prioritizing feasible solutions among the current swarm. To implement this method, the total amount of infeasibility for each particle $V\left(q_{j}\right)$ needs to be calculated. For example, if we define $\mu_{i}\left(q_{j}\right)$ as the magnitude of the particle's infeasibility for the $i$ th constraint, then $V\left(q_{j}\right)$ can be obtained via $V\left(q_{j}\right)=\sum_{i}^{N_{c}} \mu_{i}\left(q_{j}\right)$. Here, $N_{c}$ denotes the number of constraints defined in (8). The way to calculate $\mu_{i}\left(q_{j}\right)$ for the constraint $C_{i}(\cdot) \leq C_{i}^{*}$ can be written as:

$$
\mu_{i}\left(q_{j}\right)= \begin{cases}0, & C_{i}\left(q_{j}\right) \leq C_{i}^{*} ; \\ \frac{C_{i}\left(q_{j}\right)-C_{i}^{*}}{\max \left(C_{i}\left(q_{j}\right)\right)-C_{i}^{*}}, & C_{i}^{*} \leq C_{i}\left(q_{j}\right) \leq \max \left(C_{i}\left(q_{j}\right)\right) ; \\ 1, & C_{i}\left(q_{j}\right) \geq \max \left(C_{i}\left(q_{j}\right)\right) .\end{cases}
$$

Subsequently, the traditional domination relationship $\prec$ is modified by adding the rule that a particle with smaller value of $V(\cdot)$ can always dominate the particle with higher value of $V(\cdot)$.

\section{Fuzzy Adaptive Law Design}

In traditional MOPSO, the algorithm control parameters $\left[\omega, c_{1}, c_{2}\right]$ are usually assigned as constant values. However, it was analyzed in [25], [26] that a poor selection of these parameters may result in negative impacts on the evolutionary process. More precisely, a larger $\omega$, together with a larger $c_{1}$ and a smaller $c_{2}$, may result in an emphasis on the global exploration ability of the MOPSO. On the contrary, a smaller $\omega$, a smaller $c_{1}$ and a larger $c_{2}$ may result in an emphasis on the local exploitation. Therefore, in compromised point, it is desired to dynamically balance the local exploitation and global exploration during the evolution process. This eventually requires a proper treatment of the control parameters, which is fulfilled via the fuzzy adaptive law developed in this paper.

Inspired by our previous study [28], a performance metricbased fuzzy adaptive parameter tuning law is proposed by using the hypervolume (HV) and spacing (SP) information. It is worth remarking that the $\mathrm{HV}$ value is a commonly-used performance metric in multi-objective optimization. This indicator reflects both the distribution and convergence of the archive. Its value can be computed by:

$$
H V(s+1)=\operatorname{Leb}\left(\bigcup_{a \in A}\left[f_{1}(a), R_{1}\right] \times \cdots \times\left[f_{M}(a), R_{M}\right]\right)
$$

in which $\operatorname{Leb}(\cdot)$ stands for the Lebesgue measure, whereas $R=$ $\left[R_{1}, \ldots, R_{M}\right]$ represents the reference point dominated by all the particles. According to (13), $H V$ can be understood as the union of all the rectangular areas covered by the obtained pareto front. A high $H V$ value indicates that the current solution set is well-distributed. In addition, to further quantify the degree of the distribution, an SP metric is defined. Its value can be computed by:

$$
S P(s+1)=\sqrt{\frac{1}{N_{A}-1} \sum_{j=1}^{N_{A}}\left(l_{j}(s+1)-\bar{l}(s+1)\right)^{2}}
$$

where $\bar{l}(\cdot)$ stands for the average minimum Manhaton distance (MD) of all particles. The minimum MD of the $j$ th particle is denoted as $l_{j}(\cdot)$. From the definition of $S P$, a high value of $S P$ might reflect an uneven distribution of the obtained solution and vice versa.

Using the $H V$ and $S P$ metrics, the fuzzy adaptive rules are then proposed, which can be summarised in Table II. The inputs to fuzzy rules are the $\mathrm{HV}$ and SP values, while the outputs are the control parameters of MOPSO (e.g., $\omega, c_{1}$ and $c_{2}$, respectively). The proposed fuzzy rules will be applied in every generation so as to adaptively balance the exploration and exploitation. 
TABLE II: Fuzzy Adaptive Rules

\begin{tabular}{c|cc|ccc}
\hline \hline \multirow{2}{*}{ No. rule } & \multicolumn{2}{|c|}{ Input } & \multicolumn{3}{c}{ Output } \\
\cline { 2 - 6 } & $H V$ & $S P$ & $\omega$ & $c_{1}$ & $c_{2}$ \\
\hline 1 & Increase & Increase & Decrease & Decrease & Increase \\
2 & Increase & Decrease & No change & No change & No change \\
3 & Decrease & Increase & Increase & Increase & Decrease \\
4 & Decrease & Decrease & Increase & Increase & Decrease \\
\hline \hline
\end{tabular}

Fig.4 illustrates the membership functions (MFs) of the HV and SP. For simplicity reasons, these two performance indicators apply the same shape of MFs. The input to the MF is the percent change of $H V$ and $S P$ indicators, $\Delta_{H V}$ and $\Delta_{S P}$, calculated every two consecutive generations. For example, $\Delta_{H V}=\frac{H V(s+1)-H V(s)}{H V(s)}$ and $\Delta_{S P}=$ $\frac{S P(s+1)-S P(s)}{S P(s)}$, respectively. Following that, they are fuzzified to the "increase" and "decrease" membership values.

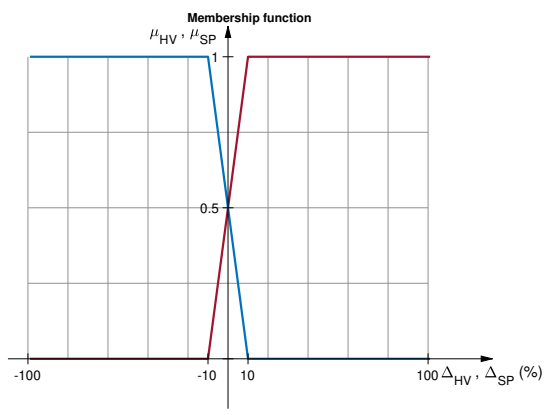

Fig. 4: Membership functions.

On the other hand, three status are defined for the fuzzy outputs (e.g., $\omega, c_{1}$ and $c_{2}$ values). That is, the "increase", "no change" and "decrease". In order to quantify the trend of these control parameters in the experiments, the following equations can be used:

$\omega(s+1)= \begin{cases}\omega(s), & \text { if } \mathrm{HV}(\mathrm{s}+1)>\mathrm{HV}(\mathrm{s}), \mathrm{SP}(\mathrm{s}+1)<\mathrm{SP}(\mathrm{s}) \\ \omega(s) \Delta_{1}(s), & \text { if } \mathrm{HV}(\mathrm{s}+1)>\mathrm{HV}(\mathrm{s}), \mathrm{SP}(\mathrm{s}+1)>\mathrm{SP}(\mathrm{s}) \\ \omega(s)\left(\Delta_{2}(s)+1\right), & \text { if } \mathrm{HV}(\mathrm{s}+1)<\mathrm{HV}(\mathrm{s}), \mathrm{SP}(\mathrm{s}+1)>\mathrm{SP}(\mathrm{s}) \\ \omega(s)\left(\frac{1}{2} \Delta_{3}(s)+1\right), & \text { if } \mathrm{HV}(\mathrm{s}+1)<\mathrm{HV}(\mathrm{s}), \mathrm{SP}(\mathrm{s}+1)<\mathrm{SP}(\mathrm{s})\end{cases}$

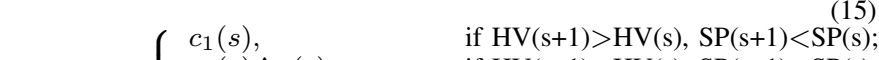

$c_{1}(s+1)= \begin{cases}c_{1}(s), & \text { if } \mathrm{HV}(\mathrm{s}+1)>\mathrm{HV}(\mathrm{s}), \mathrm{SP}(\mathrm{s}+1)>\mathrm{SP}(\mathrm{s}) ; \\ c_{1}(s) \Delta_{1}(s), & \text { if }\end{cases}$

$\begin{array}{ll}c_{1}(s)\left(\Delta_{2}(s)+1\right), & \text { if } \mathrm{HV}(\mathrm{s}+1)<\mathrm{HV}(\mathrm{s}), \mathrm{SP}(\mathrm{s}+1)>\mathrm{SP}(\mathrm{s}) ; \\ c_{1}(s)\left(\frac{1}{2} \Delta_{3}(s)+1\right), & \text { if } \mathrm{HV}(\mathrm{s}+1)<\mathrm{HV}(\mathrm{s}), \mathrm{SP}(\mathrm{s}+1)<\mathrm{SP}(\mathrm{s}) .\end{array}$

$c_{2}(s+1)= \begin{cases}c_{2}(s), & \text { if } \mathrm{HV}(\mathrm{s}+1)>\mathrm{HV}(\mathrm{s}), \mathrm{SP}(\mathrm{s}+1)<\mathrm{SP}(\mathrm{s}) \\ c_{2}(s)\left(\Delta_{1}(s)+1\right), & \text { if } \mathrm{HV}(\mathrm{s}+1)>\mathrm{HV}(\mathrm{s}), \mathrm{SP}(\mathrm{s}+1)>\mathrm{SP}(\mathrm{s}) \\ c_{2}(s)\left(\Delta_{2}(s)\right), & \text { if } \mathrm{HV}(\mathrm{s}+1)<\mathrm{HV}(\mathrm{s}), \mathrm{SP}(\mathrm{s}+1)>\mathrm{SP}(\mathrm{s}) \\ c_{2}(s)\left(\frac{1}{2} \Delta_{3}(s)\right), & \text { if } \mathrm{HV}(\mathrm{s}+1)<\mathrm{HV}(\mathrm{s}), \mathrm{SP}(\mathrm{s}+1)<\mathrm{SP}(\mathrm{s})\end{cases}$

$(16)$

\section{B. Overall Algorithm Framework}

where $\omega(s+1), c_{1}(s+1)$ and $c_{2}(s+1)$ stand for, respectively, the updated inertia weight and acceleration parameters. $\Delta_{i}(s), i=1,2,3$ is designed as:

$$
\left\{\begin{array}{l}
\Delta_{1}(s)=\max \left\{\frac{H V(s)}{H V(s+1)}, \frac{S P(s)}{S P(s+1)}\right\} \\
\Delta_{2}(s)=\max \left\{\frac{H V(s+1)}{H V(s)}, \frac{S P(s)}{S P(s+1)}\right\} \\
\Delta_{3}(s)=\min \left\{\frac{H V(s+1)}{H V(s)}, \frac{S P(s+1)}{S P(s)}\right\}
\end{array}\right.
$$

A difference can be found between the method used in [28] and the one proposed in this paper. For example, in [28], once the status of the control parameter is determined, constant increment is used to adjust the control parameters. However, in the proposed fuzzy adaptive law, the adjustment step is achieved via a fuzzy rule-based system. In this way, the emphasis on the exploration and exploitation can be smoothly adapted during the evolution process.
To provide a clear structure of the evolutionary process of the proposed FAMOPSO, key steps are summarised and presented in Algorithm 1.

Remark 2. In the proposed fuzzy adaptive rules, the worst case scenario corresponds to the fuzzy rule 3 . In this case, the obtained solutions is losing extensiveness and diversity (e.g., the pareto front is shrinking and not well-distributed). Therefore, the fuzzy adaptive law will adjust the algorithm control parameters in order to emphasize the global exploration. By contrast, if the $H V$ value is increasing and the $S P$ indicator is decreasing (e.g., fuzzy rule 2), it can be expected that the current particle swarm is uniformly distributed and converging. As a result, it is better to keep the algorithm control parameters unchanged.

\section{IMPLEMENTATION CONSIDERATION}

\section{A. Encoding and Decoding of a Particle}

In the particle encoding process, every particle is encoded as a vector representing a potential control sequence. Specifically, the control variable $u=[\eta, \omega]$ will firstly be discretized at $N_{k}$ th temporal nodes, thereby constructing the particle in $\left(2 \times N_{k}+1\right)$-dimensional space. Here, each dimension is represented by a real number and the front $2 \times N_{K}$ dimensions stand for the control sequence, whereas the last one dimension is the final time instant $t_{f}$. More precisely, the encoding style for each particle can be written as:

$$
\begin{aligned}
& \operatorname{particle}(j)=\left[\eta_{1}, \ldots, \eta_{N_{k}}, \omega_{1}, \ldots, \omega_{N_{k}}, t_{f}\right] \\
& \text { particle matrix }=\left[\operatorname{particle}(1) ; \ldots ; \operatorname{particle}\left(N_{j}\right)\right]
\end{aligned}
$$

where $N_{j}$ denotes the total number of particles.

According to the encoded particle, the position of the particle can be decoded to two parts: the vector of the control sequence and the mission time duration. Based on these pieces of information, the overtaking trajectory can then be obtained. The decoding process can be summarised as follows:

1) Implement the last dimension of the position of the particle $t_{f}$ to generate $N_{k}$ th time instant on the time domain $\left[0, t_{f}\right]$ (e.g., $\left[t_{1}, t_{2}, \ldots, t_{N_{k}}\right]$, where $\left.t_{N_{k}}=t_{f}\right)$.

2) Decode the front $2 \times N_{k}$ dimensions to the control sequence of the autonomous vehicle.

3) Construct the autonomous vehicle state trajectory: according to the temporal nodes $\left[t_{1}, t_{2}, \ldots, t_{N_{k}}\right]$ and the initial state information $x_{0}$, the equations of motion of the vehicle are integrated via numerical integration methods.

4) Output the set of autonomous vehicle state trajectory.

\section{Convergent Property of the FAMOPSO}

Following the construction of the algorithm framework, an important concern that deserves further discussion is the convergent property associated with the proposed method. Hence, we present an attempt to address this concern in this subsection.

Based on (10) and (11), it is observed that the evolutionary process of the proposed algorithm can be treated as a linear recurrence relation. That is, by taking the updated velocity equation into the updated position equation and using $q_{j, d}(s)-q_{j, d}(s-1)=v_{j, d}(s)$, we have

$$
\left[\begin{array}{c}
q_{j, d}(s+1) \\
q_{j, d}(s) \\
1
\end{array}\right]=M(s) \cdot\left[\begin{array}{c}
q_{j, d}(s) \\
q_{j, d}(s-1) \\
1
\end{array}\right]
$$




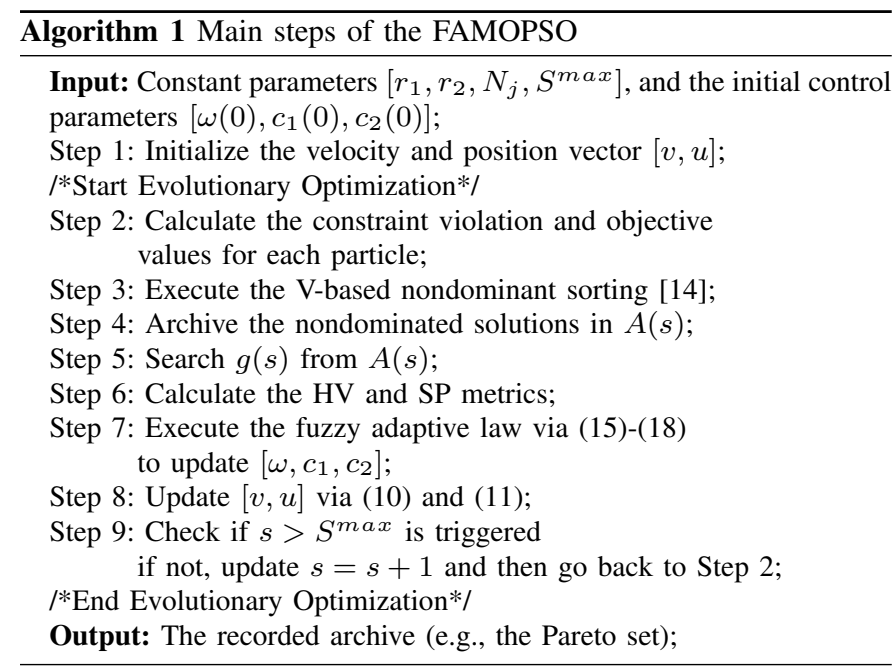

in which

$$
M=\left[\begin{array}{ccc}
1-\beta+\omega(s) & -\omega(s) & \beta_{1} p_{j, d}(s)+\beta_{2} g_{d}(s) \\
1 & 0 & 0 \\
0 & 0 & 1
\end{array}\right]
$$

In (20), $s=\{0,1,2, \ldots\} . \beta_{1}$ and $\beta_{2}$ are the abbreviations of $c_{1} r_{1}$ and $c_{2} r_{2}$, respectively. $\beta=\beta_{1}+\beta_{2}=c_{1} r_{1}+c_{2} r_{2}$. To guarantee the linear recurrence relation (20) is convergent, the following lemma [29] should be applied.

Lemma 1. Given a sequence $\left\{q_{j, d}(1), q_{j, d}(2), \ldots\right\}$ produced by (20), if the spectral radius of $M$ can satisfy $\varrho(M)=\max \left(\left|\lambda_{i}\right|\right)<1$, then the sequence $\left\{q_{j, d}(1), q_{j, d}(2), \ldots\right\}$ is convergent. Here, $\lambda_{i}$ represents the ith eigenvalue of $M$.

Lemma 1 is a necessary and sufficient condition for a convergent sequence. The study of the convergent property with respect to the proposed method will mainly rely on this lemma. Priory to discuss the main theorem, some assumptions are firstly presented.

Assumption 1. There exists a solution set $p^{*}$ for $p_{j}(s)=$ $\left[p_{j, 1}(s), \ldots, p_{j, D}(s)\right]$ and $p^{*}$ is in the sense of pareto-optimal.

Assumption 2. There exist $\beta_{1}=c_{1} r_{1}>0$ and $\beta_{2}=c_{2} r_{2}>0$ such that the parameter $\beta=\beta_{1}+\beta_{2}$ can satisfy the inequality $0<\beta<$ $2(1+\omega(s))$.

The above two assumptions, together with Lemma 1, are utilized to construct and prove the following main theorem.

Theorem 1. Define the particle position and velocity equations in the form of (10) and (11). If Assumption 1 and Assumption 2 can be satisfied, then the particle position will converge to $p^{*}$.

Proof. The entire proof contains two parts. In the first part, we show that the particle position is a convergent sequence. In the second part, we illustrate that the position will converge to $p^{*}$.

According to the recurrence relation (20) and the coefficient matrix $M$ given by (21), we can write the characteristic polynomial of (20) as:

$$
(1-\lambda)\left(\lambda^{2}-\lambda(1-\beta+\omega(s))+\omega(s)\right)=0
$$

Therefore, the three eigenvalues of $M$ are:

$$
\left\{\begin{array}{l}
\lambda_{1}=1 \\
\lambda_{2}=\left(1-\beta+\omega(s)+\sqrt{(1-\beta+\omega(s))^{2}-4 \omega(s)}\right) / 2 \\
\lambda_{3}=\left(1-\beta+\omega(s)-\sqrt{(1-\beta+\omega(s))^{2}-4 \omega(s)}\right) / 2
\end{array}\right.
$$

As a result, we can rewrite the particle position as:

$$
q_{j, d}(s)=\lambda_{1} k_{1}+\lambda_{2}^{s} k_{2}+\lambda_{3}^{s} k_{3}
$$

where $k_{1}, k_{2}, k_{3}$ are constants. From Lemma 1, it can be concluded that the evolution of particle potion will be convergent if and only if $\max \left\{\left|\lambda_{2}\right|,\left|\lambda_{3}\right|\right\}<1$. More precisely,

$$
\frac{1}{2}\left|1-\beta+\omega(s) \pm \sqrt{(1-\beta+\omega(s))^{2}-4 \omega(s)}\right|<1
$$

Now two cases should be considered: 1$)$. $\left(1-\beta+\omega(s)^{2}-4 \omega(s)\right)<0$ or 2). $\left(1-\beta+\omega(s)^{2}-4 \omega(s)\right) \geq 0$. In the first case, if the condition inequality holds true, we have

$$
\left\{\begin{array}{l}
\omega(s)>0 \\
(\omega(s)+1)-2 \sqrt{\omega(s)}<\beta<(\omega(s)+1)+2 \sqrt{\omega(s)}
\end{array}\right.
$$

Besides, from (23), $\lambda_{2}$ and $\lambda_{3}$ are two complex values. Thus

$$
\begin{aligned}
\left|\lambda_{2}\right|^{2} & =\left|\lambda_{3}\right|^{2} \\
& =\frac{1}{4}\left\|1-\beta+\omega(s) \pm \sqrt{(1-\beta+\omega(s))^{2}-4 \omega(s)}\right\|^{2} \\
& =\omega(s)
\end{aligned}
$$

Consequently, the spectral radius condition reduces to $\omega(s)<1$. Combining $\omega(s)<1$ and (26), we obtain the convergence condition for case 1):

$$
\left\{\begin{array}{l}
0<\omega(s)<1 \\
(\omega(s)+1)-2 \sqrt{\omega(s)}<\beta<(\omega(s)+1)+2 \sqrt{\omega(s)}
\end{array}\right.
$$

In terms of case 2$)$, the inequality $(1-\beta+\omega(s))^{2}-4 \omega(s) \geq 0$ will result in

$$
\left\{\begin{array}{l}
\omega(s) \geq 1 \\
\beta \leq 1-2 \sqrt{\omega(s)}+\omega(s) \quad \text { or } \quad \beta \geq 1+2 \sqrt{\omega(s)}+\omega(s)
\end{array}\right.
$$

The condition $\max \left\{\left|\lambda_{2}\right|,\left|\lambda_{3}\right|\right\}<1$ is equivalent to

$$
-1<\frac{(\omega(s)+1-\beta) \pm \sqrt{(\omega(s)+1-\beta)^{2}-4 \omega(s)}}{2}<1
$$

Since $\lambda_{2}$ and $\lambda_{3}$ are two real numbers, the equation (30) can be rewritten as

$$
\left\{\begin{array}{l}
\beta-\omega(s)-3<-\sqrt{(\omega(s)+1-\beta)^{2}-4 \omega(s)} \\
\sqrt{(\omega(s)+1-\beta)^{2}-4 \omega(s)}<1-\omega(s)+\beta
\end{array}\right.
$$

From inequality (31), we can obtain

$$
\left\{\begin{array}{l}
-3<\omega(s)+\beta<1 \\
2 \omega(s)-\beta+2>0 \\
\beta>0
\end{array}\right.
$$

Combining all the conditions for both case 1) and case 2), the overall convergence condition of the proposed method can be expressed as:

$$
\left\{\begin{array}{l}
0 \leq \omega(s)<1 \\
0<\beta<2+2 \omega(s)
\end{array}\right.
$$

According to the definitions of $\Delta_{i}(s)$ given by (18), one has

$$
\left\{\begin{array}{l}
0<\Delta_{1}(s) \leq 1 \\
0<\Delta_{2}(s) \leq 1 \\
0<\Delta_{3}(s) \leq 1
\end{array}\right.
$$

Therefore, according to Assumption 2 and (34), there exist $\omega(s)$ and $\beta$ such that the convergence condition (33) can be satisfied.

Based on the characteristic polynomial, one can obtain the convergence value of $q_{j, d}(s)$ as

$$
\lim _{s \rightarrow+\infty} q_{j, d}(s)=k_{1}
$$

To calculate $k_{1}$, we set $s=0,1,2$ in (24):

$$
\left\{\begin{array}{l}
q_{j, d}(0)=k_{1}+k_{2}+k_{3} \\
q_{j, d}(1)=k_{1}+k_{2} \lambda_{2}+k_{3} \lambda_{3} \\
q_{j, d}(2)=k_{1}+k_{2} \lambda_{2}^{2}+k_{3} \lambda_{3}^{2}
\end{array}\right.
$$


That is,

$$
\left[\begin{array}{l}
q_{j, d}(0) \\
q_{j, d}(1) \\
q_{j, d}(2)
\end{array}\right]=\left[\begin{array}{ccc}
1 & 1 & 1 \\
1 & \lambda_{2} & \lambda_{3} \\
1 & \lambda_{2}^{2} & \lambda_{3}^{2}
\end{array}\right]\left[\begin{array}{l}
k_{1} \\
k_{2} \\
k_{3}
\end{array}\right]
$$

Solving the linear system (37), the value of $k_{1}$ can be obtained as:

$$
k_{1}=\frac{\beta_{1} p_{j, d}(s)+\beta_{2} g_{d}(s)}{\beta_{1}+\beta_{2}}
$$

Based on the nondominant sorting process, we have

$$
\left\{\begin{array}{l}
p_{j, d}(s) \succ p_{j, d}(s-1) \text { or } p_{j, d}(s) \prec \succ p_{j, d}(s-1) \\
g_{d}(s) \succ p_{j, d}(s) \text { or } g_{d}(s) \prec \succ p_{j, d}(s)
\end{array}\right.
$$

Since the global best solution $g_{d}(s)$ is selected from $p_{j}(s)$, there exists $\lim _{s \rightarrow+\infty} g_{d}(s)=p^{*}$. Consequently, by defining $q_{j}(s)=$ $\left[q_{j, 1}(s), \ldots, q_{j, D}(s)\right]$, one has

$$
\lim _{s \rightarrow+\infty} q_{j}(s)=\lim _{s \rightarrow+\infty} \frac{\beta_{1} p^{*}+\beta_{2} p^{*}}{\beta_{1}+\beta_{2}}=p^{*}
$$

which completes the proof.

\section{Computational Complexity}

The computational complexity of the proposed FAMOPSO algorithm is analyzed in this subsection. In FAMOPSO, the computational complexity is mainly affected by the computation of constraints and objectives, the non-dominated sorting of the particle among the swarm and archive, and the fuzzy adaptive process. Assuming that the number of objective to be optimized is $M$, and the size of swarm and archive have the same size $N_{j}$. The computation of objectives and the constraint violation value will require $\mathcal{O}\left(M N_{j}\right)$ and $\mathcal{O}\left(N_{j}\right)$ computations, respectively. Then the non-dominated sorting process will be applied to rank the particles, which requires $\mathcal{O}\left(M N_{j}^{2}\right)$ computations [25]. In terms of the fuzzy adaptive process, the main computational burden is in the computation of the performance indicator. Specifically, to apply the fuzzy adaptive law, the $H V$ and $S P$ values should be evaluated at every generation. According to [30], the computation of $H V$ indicator requires $\mathcal{O}\left(N_{j} \log \left(N_{j}\right)+\right.$ $\left.N_{j}^{M / 2} \log \left(N_{j}\right)\right)$ operations. Compared with the calculation of $H V$, the computation of $S P$ can be negligible. Consequently, the worstcase computational complexity of one generation of the proposed FAMOPSO is $\mathcal{O}\left(M N_{j}^{2}\right)$.

\section{Results And Discussions}

In this section, testing on simulations of different overtaking scenarios is executed to illustrate the implementation of the FAMOPSO algorithm as well as to analyze the trade-off between minimizing time duration, path smoothness, and maximizing visibility.

\section{A. Parameter Assignment}

Some vehicle/mission-related parameters [24], together with the control variables of the proposed FAMOPSO algorithm, are firstly specified. These parameters/variables will be used to calculate the multi-objective optimal overtaking maneuver results and their values/ranges are tabulated in Table III.

Next, the positional data for different on-road obstacles $O^{p}, p=$ 1,2 is given below:

$$
O^{1}\left\{\begin{array} { l } 
{ A _ { x } = 2 5 . 0 1 , A _ { y } = 3 . 0 1 } \\
{ B _ { x } = 2 8 . 6 6 , B _ { y } = 4 . 6 4 } \\
{ C _ { x } = 2 7 . 9 4 , C _ { y } = 6 . 2 5 } \\
{ D _ { x } = 2 4 . 2 9 , D _ { y } = 4 . 6 3 }
\end{array} \quad O ^ { 2 } \left\{\begin{array}{l}
A_{x}=7.32, A_{y}=1.81 \\
B_{x}=10.92, B_{y}=0.06 \\
C_{x}=11.69, C_{y}=1.65 \\
D_{x}=8.10, D_{y}=3.40
\end{array}\right.\right.
$$

In addition, the vehicle starts the maneuver phase from a normal driving condition which is defined by $\left[p_{x}(0), p_{y}(0), v(0), a(0), \theta(0), \phi(0)\right]=\left[0.7 \mathrm{~m}, 4.5 \mathrm{~m}, 5 \mathrm{~m} / \mathrm{s}, 0 \mathrm{~m} / \mathrm{s}^{2}\right.$,
TABLE III: Specification of different parameters/variables

\begin{tabular}{lclc}
\hline \hline Parameters & Values & Variables & Ranges \\
\hline$n$ & 0.8 & $p_{x}$ & {$[0,50]$} \\
$l$ & 2.5 & $p_{y}$ & {$[0,6]$} \\
$C L$ & 6.0 & $v$ & {$[-10,10]$} \\
$b$ & 0.8855 & $a$ & {$[-0.75,0.75]$} \\
$m$ & 0.7 & $\theta$ & {$\left[-90^{\circ}, 90^{\circ}\right]$} \\
$\omega(0)$ & $\left(1+r_{1}\right) / 2$ & $\phi$ & {$\left[-33^{\circ}, 33^{\circ}\right]$} \\
$c_{1}(0)$ & 1.49445 & $r_{1}$ & {$[0,1]$} \\
$c_{2}(0)$ & 1.49445 & $r_{2}$ & {$[0,1]$} \\
$N_{k}$ & 60 & $c_{1}$ & {$[1,2]$} \\
$N_{j}$ & 100 & $c_{2}$ & {$[1,2]$} \\
$S^{\text {max }}$ & 200 & $t$ & {$[0,20]$} \\
\hline \hline
\end{tabular}

$0^{\circ}, 0^{\circ}$. Once the overtaking is completed, the terminal condition specified by (7) should be satisfied. Two additional control path constraints in terms of the jerk variable $\eta$ as well as the curvature derivative $\dot{k}$ are given by $\eta \in[-2,2]$ and $\dot{k} \in[-0.6,0.6]$, respectively. After specifying all the variables and constraints, the multi-objective overtaking problem is solved via the proposed FAMOPSO algorithm.

\section{B. Multi-Objective Optimal Overtaking Results}

In this subsection, the multi-objective optimal overtaking maneuver results obtained by applying the proposed FAMOPSO method are displayed and the trade-off between different mission objectives is analyzed. The obtained pareto front is presented in Fig.5 and projected onto two planes: minimizing time duration versus maximizing the visibility, and minimizing time duration versus minimizing path smoothness. It is worth noting that all the solutions among the final solution set have zero constraint violation values. This guarantees the effectiveness of the obtained solution, which is also a prerequisite for the validity of a multi-objective autonomous vehicle maneuver planning approach.
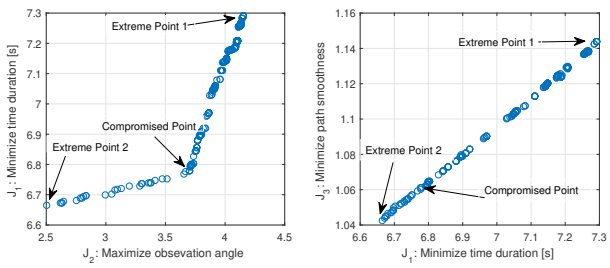

Fig. 5: Final pareto set results.

From Fig.5, it is obvious that minimizing the mission time and maximizing the visibility are two contradicting objectives. This is because if the mission planner wants to prioritize mission time minimization, a more aggressive overtaking maneuver might be found from the solution. That is, the vehicle tends to overtake the on-road object using a relatively-narrow corridor. By contrast, if it is desired to fulfill the mission with visibility maximization, the autonomous car may have to move significantly into the opposite lane in order to avoid collision with the on-road obstacle as well as cleanly see in front of a car ahead. However, this will inevitably increase the time duration. Interestingly, a highly-correlated relationship can be observed between minimizing the mission time duration and minimizing the path smoothness. This can be explained that according to (2), the smoothness indicator value is largely depended on the upper limit of integration $\left(t_{f}\right)$. Besides, since minimizing time duration may result in a narrow driving corridor, the path smoothness indicator may also be decreased.

Based on the generated pareto front, two extreme solutions, along with one compromised solution, can be detected (as indicated 
in Fig.5). The objective and the constraint violation values of these three solutions are tabulated in Table IV.

TABLE IV: Extreme and compromised solutions

\begin{tabular}{lcccc}
\hline \hline Different & \multicolumn{3}{c}{ Objectives } & Constraint violation \\
\cline { 2 - 4 } solutions & $J_{1}$ & $J_{2}$ & $J_{3}$ & $V$ \\
\hline Extreme point 1 $p_{2}$ & 7.2908 & 4.1505 & 1.1437 & 0 \\
Extreme point 2 $p_{1}$ & 6.6639 & 2.5008 & 1.0426 & 0 \\
Compromised point $p_{i}^{*}$ & 6.7774 & 3.6666 & 1.0601 & 0 \\
\hline \hline
\end{tabular}

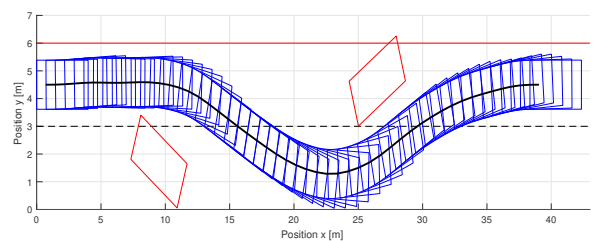

Fig. 6: The overtaking maneuver profile: Extreme point 1.
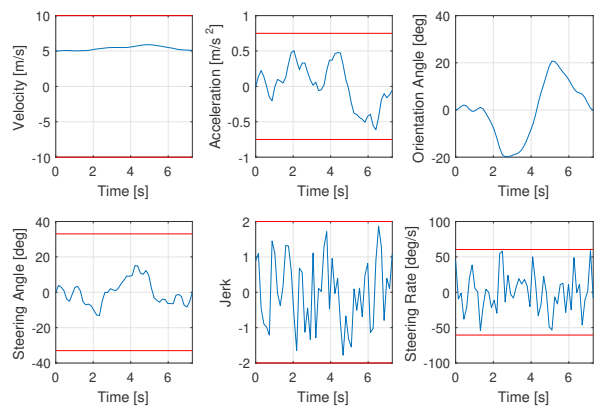

Fig. 7: Optimized state/control trajectories: Extreme point 1.

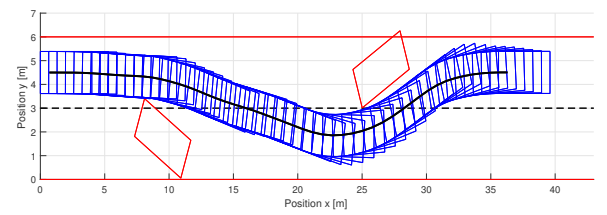

Fig. 8: The overtaking maneuver profile: Extreme point 2.
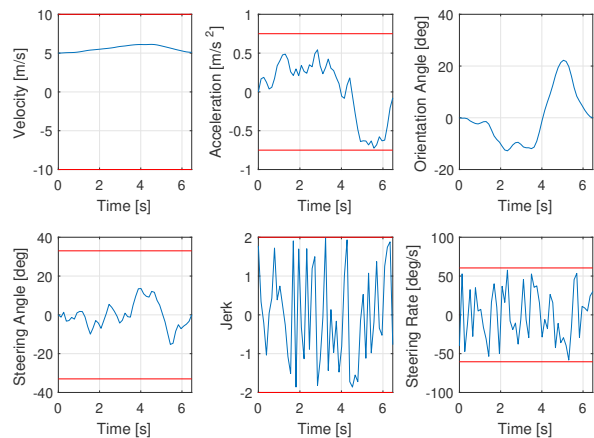

Fig. 9: Optimized state/control trajectories: Extreme point 2.

From Table IV, extreme point 1 can be treated as the solution with the best overtaking visibility but the worst overtaking time and path smoothness. The corresponding overtaking maneuver profile is depicted in Fig.6, while the optimized state/control trajectories are

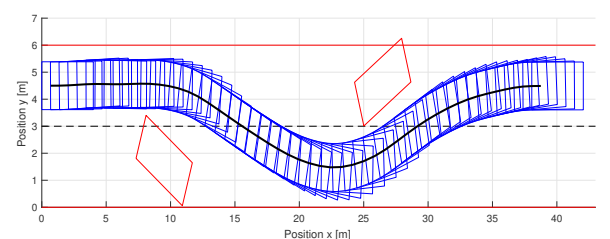

Fig. 10: Overtaking maneuver profile: Compromised point.
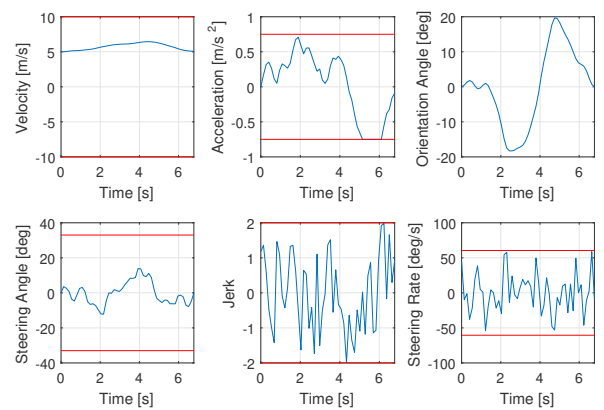

Fig. 11: Optimized state/control trajectories: Compromised point.

plotted in Fig.7. On the other hand, extreme point 2 represents the solution where the overtaking time and path smoothness can be maximally optimized at the expense of overtaking visibility. The optimal overtaking maneuver result for this extreme solution is presented in Fig.8, whereas the corresponding optimal state/control profiles are compressed in Fig.9.

It should be noted that the best compromised solution is obtained by searching the candidate among the final pareto set. Specifically, we are interested in finding a pareto-optimal solution that the total goal attainment values of the three mission objectives can be maximized. The goal attainment value for each mission objective can be calculated by measuring the magnitude of achieving its extreme value. Take the objective $J_{1}$ as an instance, the goal attainment value $\mu_{J_{1}} \in[0,1]$ is calculated via $\mu_{J_{1}}=1-\frac{J_{1}\left(p_{i}\right)-J_{1}^{*}}{J_{1}^{m a x}-J_{1}^{*}}$, where $p_{i} \in P$ is the candidate solution on the obtained front $P$. Here, the extreme value of $J_{1}$ can be set to $J_{1}^{*}=6.6639$ and $J_{1}^{\max }=7.2908$. The goal attainment values with respect to $J_{2}$ and $J_{3}$ can be computed analogically. After calculating the goal attainment value for each point among the pareto-optimal set, the best compromised solution listed in Table IV is obtained by performing

$$
p_{i}^{*}=\arg \max _{p_{i} \in P} \sum_{i=1}^{3} \mu_{J_{i}}
$$

In this way, the solution with the maximum total goal achievement value can be selected. The compromised overtaking maneuver profile, together with the compromised state/control trajectories, can be found in Fig.10 and Fig.11, respectively. All these results provided earlier confirm that it is able to use the proposed method to produce an overtaking maneuver in the pareto-optimal sense while prioritizing safety and respecting the boundaries of the road.

It is worth noting that in the simulation, limited computation efforts (as specified in Table Table III) are used for the proposed algorithm. Besides, the implementation of bio-inspired optimization algorithms will inevitably introduce randomness in the optimization process. Due to these reasons, oscillations may be detected from the trajectory results shown in Fig.7, Fig.9 and Fig.11. Based on our experiments, this undesired phenomenon can be alleviated if higher computation efforts are used. For example, by increasing the computing power, a more aggressive extreme point 2 can be obtained 
with a relatively-obvious bang-bang control structure.

\section{Comparison Against Other Optimization Methods}

In the previous subsection, we have shown that it is effective to apply the proposed FAMOPSO algorithm for planning the multiobjective optimal overtaking maneuver of the autonomous ground vehicle. A best compromised solution is found based on the produced pareto-optimal set. In this subsection, we perform the comparative study for different overtaking scenarios so as to illustrate the advantage and superiority of applying the designed method.

Two overtaking mission scenarios are firstly constructed for the purpose of comparison:

- Case 1: Overtaking object 1 while optimizing $\left[J_{1}, J_{2}, J_{3}\right]$.

- Case 2: Overtaking object 1 and object 2 while optimizing $\left[J_{1}, J_{2}, J_{3}\right]$.

Firstly, comparative studies were performed between the proposed FAMOPSO and other state-of-the-art evolutionary multiobjective optimization (EMO) methods. For example, a modified NSGA-II (MNSGA-II) algorithm proposed in [31], an enhanced MOEA/D-DE method investigated in [32], and an improved multiobjective artificial bee colony (I-MOABC) developed in [20]. Different from the traditional NSGA-II algorithm, a well-distributed set of reference point and a new diversity factor were adopted in the MNSGA-II method in order to avoid the premature convergence. Besides, in the MOEA/D-DE algorithm, a uniform weight-vector distribution strategy was applied to further guide the optimization process [32]. Regarding the computational complexity of the different EMOs, the worst-case computational complexity for the MNSGA-II algorithm is $\mathcal{O}\left((M+1) N_{j}^{2}\right)$ [31], whereas the I-MOABC method requires $\mathcal{O}\left(M N_{j}^{2}\right)$ computations for one generation [33]. As for the MOEA/D-DE, the original multi-objective problem is decomposed into a number of single-objective problems (SOPs). Then the algorithm aims to optimize these SOPs simultaneously [32]. One advantage of using MOEA/D-DE is that the computational complexity is smaller than other methods tested in the paper.

By applying different algorithms, the pareto front solutions for Case 1 and Case 2 are displayed in Fig.12 and Fig.13, respectively. From the pareto results, it is obvious that the proposed FAMOPSO can generally perform better than other methods tested in this paper for the two mission cases. This can be reflected by the fact that the final pareto set computed using the FAMOPSO can dominate the solution calculated using other EMOs studied in this paper. In addition, the distribution of the pareto front produced by FAMOPSO tends to be more uniform than the results calculated by using other techniques. This can be attributed to the implementation of the designed fuzzy adaptive law which balances the local exploitation and global exploration of the optimization process, thus making the pareto set more optimal.
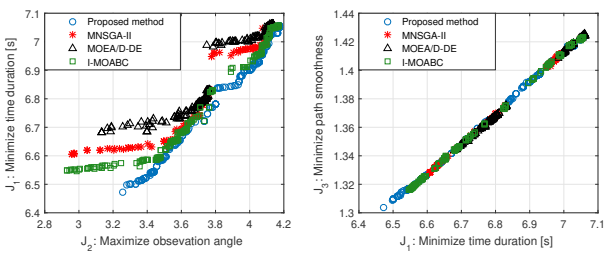

Fig. 12: Pareto front solutions for Case 1.

To offer a quantitative analysis, the HV performance metric is applied as an indicator to evaluate the quality of the pareto solution calculated via different multi-objective optimization methods (e.g., the population diversity as well as the distribution uniformity). The
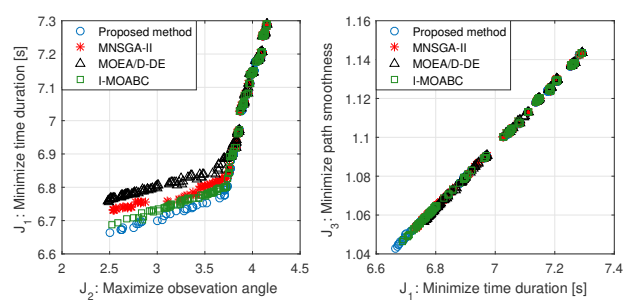

Fig. 13: Pareto front solutions for Case 2.

HV results for the two overtaking mission cases obtained via different BIO-based methods are tabulated in Table V and Table VI, where the reference point information used to calculate the HV metric is assigned as $R=[8,2,1.5]$. Note that a different selection of the reference point might result in a difference with respect to the calculated HV value.

TABLE V: HV results (Case 1)

\begin{tabular}{c|ccc}
\hline \hline & \multicolumn{3}{|c}{ HV } \\
\hline Case 1 & Best & Average & Worst \\
\hline Proposed & $\mathbf{0 . 5 4 7 4}$ & $\mathbf{0 . 5 4 6 1}$ & $\mathbf{0 . 5 4 4 6}$ \\
MNSGA-II [31] & 0.4443 & 0.4431 & 0.4424 \\
MOEA/D-DE [32] & 0.3873 & 0.3861 & 0.3855 \\
I-MOABC [20] & 0.4937 & 0.4927 & 0.4919 \\
\hline \hline
\end{tabular}

TABLE VI: HV results (Case 2)

\begin{tabular}{c|ccc}
\hline \hline & \multicolumn{3}{|c}{ HV } \\
\hline Case 1 & Best & Average & Worst \\
\hline Proposed & $\mathbf{1 . 1 6 7 7}$ & $\mathbf{1 . 1 6 5 3}$ & $\mathbf{1 . 1 6 3 6}$ \\
MNSGA-II [31] & 1.1072 & 1.1053 & 1.1035 \\
MOEA/D-DE [32] & 1.0681 & 1.0666 & 1.0652 \\
I-MOABC [20] & 1.1443 & 1.1404 & 1.1374 \\
\hline \hline
\end{tabular}

As discussed in Section III.C, a high $H V$ value reflects the obtained solution set is well-converged and well-distributed. Based on the data presented in Table V and Table VI, it can be observed that the FAMOPSO algorithm is able to produce the highest HV results. The value of the HV indicator truly reflects the pareto front results obtained using different methods shown in Fig.12 and Fig.13. That is, the pareto front obtained via other methods can generally be covered by the front calculated via the proposed method. These results further confirm that the proposed strategy can be applied as a more effective alternative to produce multi-objective optimal overtaking maneuver for the car-like autonomous vehicle.

Furthermore, we also give attention to the performance between the proposed FAMOPSO and other MOPSO-based methods. A PSObased autonomous vehicle motion planner was developed in [24]. This method was shown to be effective for planning the movement of the autonomous vehicle and is extended to a multi-objective version for the purpose of comparison (denoted as MOPSO). It should be noted that the main difference between the proposed FAMOPSO and the MOPSO lies in the use of the fuzzy adaptive component. In addition, it is necessary to show the superiority of the proposed method in comparison with some existing FAMOPSO methods. For instance, a best fitness-based FAMOPSO method proposed in [34] (denoted as bf-FAMOPSO). Mission case 1 was re-performed by applying the three MOPSO-based methods and the HV results are presented in Table VII, from where it can be seen that the proposed method can achieve the highest HV result. Moreover, the bf-FAMOPSO can outperform the basic MOPSO.

The result presented in Table VII confirms that it is advantageous to apply the fuzzy adaptive component to adjust the evolution process of the MOPSO method. Besides, the proposed fuzzy adaptive law can 
TABLE VII: Performance of different MOPSO-based methods

\begin{tabular}{c|ccc}
\hline \hline & \multicolumn{3}{|c}{ HV } \\
\hline Case 1 & Proposed & MOPSO [24] & bf-FAMOPSO [34] \\
\hline Best & $\mathbf{0 . 5 4 7 4}$ & 0.3316 & 0.4729 \\
Average & $\mathbf{0 . 5 4 6 1}$ & 0.3307 & 0.4718 \\
Worst & $\mathbf{0 . 5 4 4 6}$ & 0.3301 & 0.4708 \\
\hline \hline
\end{tabular}

perform better than the fuzzy adaptive law proposed in [34] in terms of achieving faster convergence and better-distributed pareto front for the automatic overtaking problem.

\section{Study on System Parameters of the Algorithm}

It should be noted that one important process that could have significant influence on the optimal result is the selection of system parameters of the algorithm. For example, the number of temporal nodes $N_{k}$, the number of particles among the swarm, and the number of generations $S^{\max }$.

In Table II, we fix the values for $\left[N_{k}, N_{j}, S^{\max }\right]$ to $[60,100,1000]$. This parameter setting is determined by performing the design of experiment (DOE) method. A similar implementation of the DOE method in terms of determining the algorithm parameters can also be found in [20]. To apply the DOE, each parameter is specified by four levels (e.g., as indicated in Table VIII), thereby resulting in an orthogonal array shown in Table IX. The proposed algorithm is independently run 20 times for each parameter combination. The obtained response HV values of different parameter combinations are summarised in Table IX, whereas the level trends of these three parameters are depicted in Fig.14.

TABLE VIII: Parameter combinations

\begin{tabular}{ccccc}
\hline \hline Parameter & \multicolumn{4}{c}{ Level } \\
\cline { 2 - 5 } & 1 & 2 & 3 & 4 \\
\hline$N_{k}$ & 20 & 40 & 60 & 80 \\
$N_{j}$ & 20 & 50 & 100 & 150 \\
$S^{\text {max }}$ & 200 & 500 & 1000 & 1500 \\
\hline \hline
\end{tabular}

TABLE IX: Results of different parameter combinations

\begin{tabular}{ccccc}
\hline \hline Experiment & \multicolumn{3}{c}{ Level } & Result \\
\cline { 2 - 4 } & $N_{k}$ & $N_{j}$ & $S^{\text {max }}$ & $H V$ \\
\hline No.1 & 1 & 1 & 1 & 0.2638 \\
No.2 & 1 & 2 & 2 & 0.2841 \\
No.3 & 1 & 3 & 3 & 0.3433 \\
No.4 & 1 & 4 & 4 & 0.3502 \\
No.5 & 2 & 1 & 2 & 0.3772 \\
No.6 & 2 & 2 & 1 & 0.3843 \\
No.7 & 2 & 3 & 4 & 0.4141 \\
No.8 & 2 & 4 & 3 & 0.4389 \\
No.9 & 3 & 1 & 3 & 0.5106 \\
No.10 & 3 & 2 & 4 & 0.5469 \\
No.11 & 3 & 3 & 1 & 0.5317 \\
No.12 & 3 & 4 & 2 & 0.5255 \\
No.13 & 4 & 1 & 4 & 0.4658 \\
No.14 & 4 & 2 & 3 & 0.4886 \\
No.15 & 4 & 3 & 2 & 0.5029 \\
No.16 & 4 & 4 & 1 & 0.4244 \\
\hline \hline
\end{tabular}

From Fig.14, we can observe that the proposed method tends to have a better performance if the parameter specification $\left[N_{k}, N_{j}, S^{\max }\right]=[60,100,1000]$ can be applied (e.g., $N_{k}$ of level $3, N_{j}$ of level 3, and $S^{\max }$ of level 3). In addition, it is also obvious that compared with $N_{j}$ and $S^{\max }$, the selection of $N_{k}$ tends to be more critical. This further confirms the necessity of applying the DOE method to determine the system parameter of the proposed algorithm.

\section{E. Impact of the Constraint Modelling Strategy}

In this subsection, the impact of the collision-free constraint modelling strategy on the pareto results and algorithm convergence
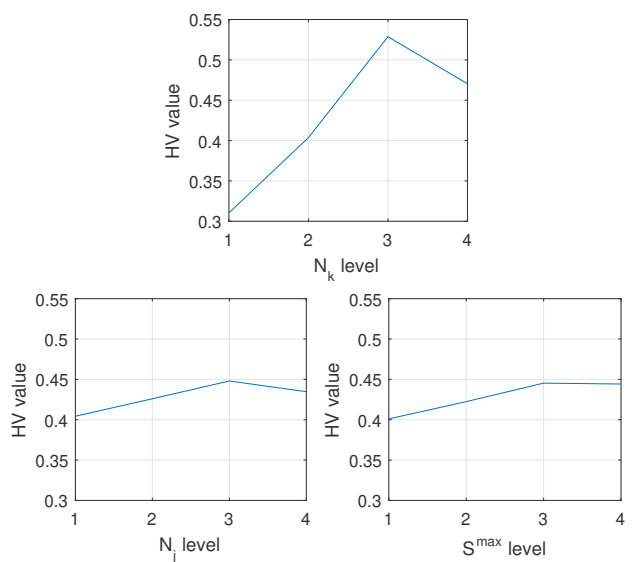

Fig. 14: Parameter level trends.

ability is studied. More precisely, the corner-point-based and the distance-based collision-free constraint modelling methods discussed in Section II.C are embedded in the FAMOPSO, MNSGA-II, IMOABC and MOEA/D-DE algorithms. Subsequently, the two overtaking scenarios were further executed.

Based on the experiment, it is found that for the optimal overtaking scenarios considered in this research, algorithms using the distance-based collision-free constraint modelling method cannot produce effective maneuver profiles for the autonomous vehicle. Following a large number of optimization iterations, all the EMOs still failed to satisfy the distance-based collision-free constraints (e.g., the constraint violation values for individuals are not zero). An important reason is that the use of distance-based obstacle avoidance strategy might introduce large conservatism, thereby restricting the convergence ability of the optimization algorithm.

By contrast, the feasible set regulated by the corner-point-based modelling strategy tends to be larger than the one defined by the distance-based method. As a result, algorithms using the cornerpoint-based collision-free constraint modelling strategy are likely to be less conservative. This indicates that the pareto solution can be better explored, as the feasible region becomes larger. Hence, it can be concluded that it is more advantageous to apply the cornerpoint-based constraint modelling strategy for addressing the optimal overtaking scenario researched in this paper.

\section{F. Impact of the Vehicle-Related Parameter}

In previous subsections, the proposed method is shown optimal in optimally solving the multi-objective overtaking trajectory planning problem. This subsection studies how the parameter variations of autonomous ground vehicles affect the final results. It should be noted that in practice, the physical constraints of the vehicle state variable (3) might not be perfectly modeled. Therefore, an uncertain assessment is performed with respect to the magnitude of the vehicle's physical constraints.

Take overtaking case 1 as an instance, it is assumed that there are some variations with respect to the tolerance region of the vehicle state variables (e.g., $5 \%$ or $10 \%$ tightness). The resulting paretooptimal result is shown in Fig. 15.

From the result presented in Fig.15, it can be seen that the obtained pareto-optimal result slightly moves away from the nominal solutions. However, the difference is not significant. Hence, it can be concluded that the proposed method is generally robust against the parameter variations of autonomous ground vehicles. 

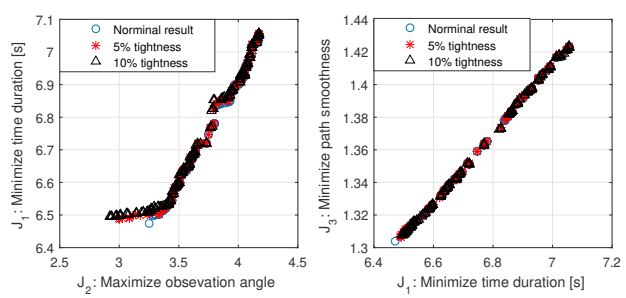

Fig. 15: Pareto results with parameter variations.

\section{G. Online Performance of the Proposed Motion Planner}

In this subsection, the possibility of extending the proposed multi-objective motion planner to real-time applications is investigated. From the off-line design and analysis, we know that optimizing $J_{1}$ and $J_{2}$ are two contradicting objectives, while optimizing $J_{1}$ and $J_{3}$ are two highly-correlated objectives. Therefore, three test scenarios are built for analyzing the performance of the online replanning process:

- Scenario 1: We aim to achieve the overtaking with optimal $J_{1}$ and $J_{3}$.

- Scenario 2: We aim to achieve the overtaking with optimal $J_{2}$.

- Scenario 3: We aim to achieve the overtaking with a compromised $J_{1}, J_{2}$ and $J_{3}$.

To achieve the real-time capability, an on-site initialization strategy is firstly applied. That is, the optimal solutions obtained via the offline experiments are recorded in a dataset. These solutions will then be applied to form the initial swarm so as to "warmly trigger" the evolutionary process of the proposed algorithm. Following that, we can reduce the computing power of the proposed method to a relatively-small scale (e.g., $\left[N_{j}, S^{\max }\right]=[20,15]$ ) and use it as a near-optimal solution generator in the online process. Significant reduction with respect to the computation time can be achieved if an optimal control solver can start its solution-finding process ar a near-optimal solution (e.g., solutions shown in Fig.5). This conclusion was validated in the previous work [8]. Hence, we embed the on-site initialization strategy and the proposed method in the optimal control solver developed in [7], thereby creating an online replanning scheme to react to potential errors/mismatches that usually exist in a real-world environment. This scheme can be treated as a receding horizon replanning procedure. That is, at each time instant, the constructed scheme produces the control command for the next control horizon. Subsequently, the autonomous vehicle executes the overtaking maneuver using these control commands until the end of this control horizon. This recursive process will continue until the terminal condition of the mission is triggered.

The online maneuver profiles of the vehicle for the three test scenarios are displayed in Fig.16. According to the results, it is obvious that the real-time maneuver solution slightly diverges from the offline pre-planned solution. However, the online replanning process is still able to maintain the trend of the pre-planned optimal results and produce feasible maneuver profile for the autonomous vehicle.

In addition, detailed results about the receding horizon replanning process are depicted in Fig.17, where the red pillars represent the length of control horizon, whereas the blue pillars indicate the average online computation duration for different test scenarios. From Fig.17, it can be observed that the replanning procedure can be accomplished before the control horizon terminates. This result, together with the obtained maneuver profiles, can increase the trust on the online use of the propose strategy. However, it is undeniable that the processing time required for the re-optimization process is hard to predict when more complicated traffic environment is considered. In this case,
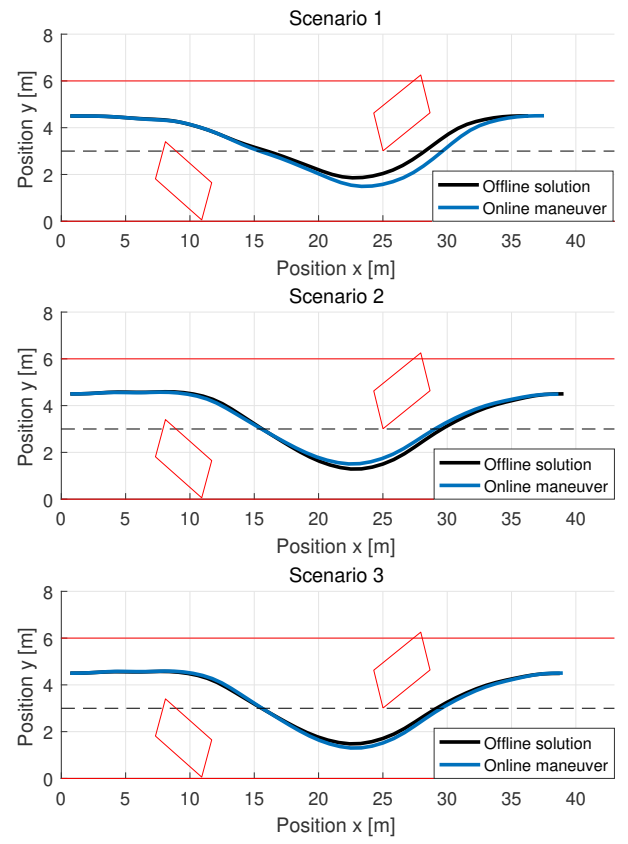

Fig. 16: Online maneuver profiles for the three test scenarios.

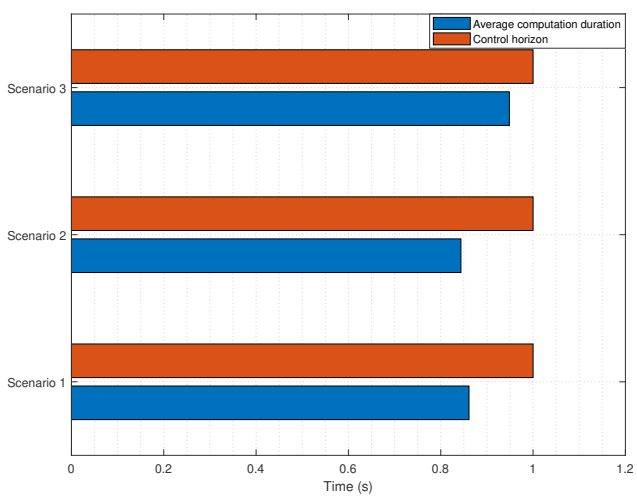

Fig. 17: Detailed results about the replanning process.

the control performance tends to be sacrificed if the re-optimization problem is insufficiently solved. Actually, if parallel computing can be utilized to optimize the control command, the online processing ability and control performance might be further improved.

\section{CONCLUding REMARK}

In this paper, the problem of overtaking irregularly-placed onroad objects for the autonomous ground vehicle was considered. An FAMOPSO algorithm was designed and utilized to produce the multi-objective optimal overtaking maneuver for the vehicle with the consideration of different physical constraints. In order to effectively balance the local exploitation and global exploration of the optimization process, a fuzzy adaptive law was constructed to dynamically adjust control parameters of the algorithm. A number of simulation studies were executed to validate the effectiveness of the optimal overtaking results as well as the enhanced performance of the designed FAMOPSO approach. Based on the obtained results, we have gained a better understanding of the trade-off between mission 
objectives. The best compromised solution, where the total goal attainment value of the three mission objectives can be maximized while prioritizing safety and respecting the boundaries of the road, has been found via the proposed method. Moreover, according to the comparative study, the designed FAMOPSO algorithm can be superior in quality and distribution of the pareto solution to other typical multi-objective optimization techniques investigated in this study.

The work presented in this paper can be extended in multiple directions. One potential direction is to extend the current problem formulation to more complex automatic overtaking scenarios. For example, we can model the obstacle to be overtaken as a dynamic object, and this will certainly bring more challenges to the constraint handling process. Moreover, another potential direction could be applying the proposed FAMOPSO algorithm to address more realworld engineering optimization problems, such as the multi-objective vehicle routing problem [15], [21], the multi-agent path planning problem [35], etc.

\section{REFERENCES}

[1] A. Macwan, J. Vilela, G. Nejat, and B. Benhabib, "A multirobot pathplanning strategy for autonomous wilderness search and rescue," IEEE Transactions on Cybernetics, vol. 45, no. 9, pp. 1784-1797, 2015.

[2] Y. Wang, S. Wang, M. Tan, C. Zhou, and Q. Wei, "Real-time dynamic dubins-helix method for 3-d trajectory smoothing," IEEE Transactions on Control Systems Technology, vol. 23, no. 2, pp. 730-736, 2015.

[3] C. Sun, Y.-C. Liu, R. Dai, and D. Grymin, "Two approaches for path planning of unmanned aerial vehicles with avoidance zones," Journal of Guidance, Control, and Dynamics, vol. 40, no. 8, pp. 2076-2083, 2017.

[4] F. You, R. Zhang, G. Lie, H. Wang, H. Wen, and J. Xu, "Trajectory planning and tracking control for autonomous lane change maneuver based on the cooperative vehicle infrastructure system," Expert Systems with Applications, vol. 42, no. 14, pp. 5932-5946, 2015.

[5] T. Mercy, R. V. Parys, and G. Pipeleers, "Spline-based motion planning for autonomous guided vehicles in a dynamic environment," IEEE Transactions on Control Systems Technology, vol. 26, no. 6, pp. 21822189, 2018.

[6] P. Narayan, P. Meyer, and D. Campbell, "Embedding human expert cognition into autonomous uas trajectory planning," IEEE Transactions on Cybernetics, vol. 43, no. 2, pp. 530-543, 2013.

[7] R. Chai, A. Savvaris, A. Tsourdos, S. Chai, and Y. Xia, "Trajectory optimization of space maneuver vehicle using a hybrid optimal control solver," IEEE Transactions on Cybernetics, vol. 49, no. 2, pp. 467-480, 2019.

[8] R. Chai, A. Savvaris, and A. Tsourdos, "Violation learning differential evolution-based hp-adaptive pseudospectral method for trajectory optimization of space maneuver vehicle," IEEE Transactions on Aerospace and Electronic Systems, vol. 53, no. 4, pp. 2031-2044, 2017.

[9] R. Chai, A. Savvaris, A. Tsourdos, S. Chai, and Y. Xia, "Improved gradient-based algorithm for solving aeroassisted vehicle trajectory optimization problems," Journal of Guidance, Control, and Dynamics, vol. 40, no. 8, pp. 2093-2101, 2017.

[10] B. Li, K. Wang, and Z. Shao, "Time-optimal maneuver planning in automatic parallel parking using a simultaneous dynamic optimization approach," IEEE Transactions on Intelligent Transportation Systems, vol. 17, no. 11 , pp. 3263-3274, 2016.

[11] B. Li and Z. Shao, "Precise trajectory optimization for articulated wheeled vehicles in cluttered environments," Advances in Engineering Software, vol. 92, pp. 40-47, 2016.

[12] H. Gao, X. Yang, and P. Shi, "Multi-objective robust h-infinity control of spacecraft rendezvous," IEEE Transactions on Control Systems Technology, vol. 17, no. 4, pp. 794-802, 2009.

[13] G.-P. Liu, J.-B. Yang, and J.-F. Whidborne, Multiobjective Optimisation and Control. Engineering Systems Modeling and Control Series, 2003.

[14] R. Chai, A. Savvaris, A. Tsourdos, Y. Xia, and S. Chai, "Solving multiobjective constrained trajectory optimization problem by an extended evolutionary algorithm," IEEE Transactions on Cybernetics, to be published.

[15] J. Wang, Y. Zhou, Y. Wang, J. Zhang, C. L. P. Chen, and Z. Zheng, "Multiobjective vehicle routing problems with simultaneous delivery and pickup and time windows: Formulation, instances, and algorithms," IEEE Transactions on Cybernetics, vol. 46, no. 3, pp. 582-594, 2016.
[16] B. A. Conway, "A survey of methods available for the numerical optimization of continuous dynamic systems," Journal of Optimization Theory and Applications, vol. 152, no. 2, pp. 271-306, 2012.

[17] B. Ji, X. Yuan, and Y. Yuan, "Modified nsga-ii for solving continuous berth allocation problem: Using multiobjective constraint-handling strategy," IEEE Transactions on Cybernetics, vol. 47, no. 9, pp. 2885-2895, 2017.

[18] R. Chai, A. Savvaris, A. Tsourdos, S. Chai, and Y. Xia, "Unified multiobjective optimization scheme for aeroassisted vehicle trajectory planning," Journal of Guidance, Control, and Dynamics, vol. 41, no. 7, pp. 1521-1530, 2018.

[19] J.-Q. Li, Q.-K. Pan, and M. F. Tasgetiren, "A discrete artificial bee colony algorithm for the multi-objective flexible job-shop scheduling problem with maintenance activities," Applied Mathematical Modelling, vol. 38, no. 3, pp. 1111-1132, 2014.

[20] J.-Q. Li, S.-C. Bai, P.-Y. Duan, H.-Y. Sang, Y.-Y. Han, and Z.-X. Zheng, "An improved artificial bee colony algorithm for addressing distributed flow shop with distance coefficient in a prefabricated system," International Journal of Production Research, pp. 1-21, 2019.

[21] Y. Fu, M. Ding, C. Zhou, and H. Hu, "Route planning for unmanned aerial vehicle (uav) on the sea using hybrid differential evolution and quantum-behaved particle swarm optimization," IEEE Transactions on Systems, Man, and Cybernetics: Systems, vol. 43, no. 6, pp. 1451-1465, 2013.

[22] Y. Tang, H. Gao, J. Kurths, and J. Fang, "Evolutionary pinning control and its application in uav coordination," IEEE Transactions on Industrial Informatics, vol. 8, no. 4, pp. 828-838, 2012.

[23] V. Roberge, M. Tarbouchi, and G. Labonte, "Comparison of parallel genetic algorithm and particle swarm optimization for real-time uav path planning," IEEE Transactions on Industrial Informatics, vol. 9, no. 1, pp. $132-141,2013$.

[24] R. Chai, A. Tsourdos, A. Savvaris, S. Chai, and Y. Xia, "Two-stage trajectory optimization for autonomous ground vehicles parking maneuver," IEEE Transactions on Industrial Informatics, to be published.

[25] Q. Zhu, Q. Lin, W. Chen, K. C. Wong, C. A. C. Coello, J. Li, J. Chen, and J. Zhang, "An external archive-guided multiobjective particle swarm optimization algorithm," IEEE Transactions on Cybernetics, vol. 47, no. 9, pp. 2794-2808, 2017.

[26] H. Han, W. Lu, and J. Qiao, "An adaptive multiobjective particle swarm optimization based on multiple adaptive methods," IEEE Transactions on Cybernetics, vol. 47, no. 9, pp. 2754-2767, 2017.

[27] F. Mohseni, J. Aslund, E. Frisk, and L. Nielsen, "Fuel and comfort efficient cooperative control for autonomous vehicles," in 2017 IEEE Intelligent Vehicles Symposium (IV), Conference Proceedings, pp. 16311636.

[28] R. Chai, A. Savvaris, A. Tsourdos, and S. Chai, "Multi-objective trajectory optimization of space manoeuvre vehicle using adaptive differential evolution and modified game theory," Acta Astronautica, vol. 136, pp. 273-280, 2017.

[29] D. Boley, "Local linear convergence of the alternating direction method of multipliers on quadratic or linear programs," SIAM Journal on Optimization, vol. 23, no. 4, pp. 2183-2207, 2013.

[30] N. Beume, C. M. Fonseca, M. Lopez-Ibanez, L. Paquete, and J. Vahrenhold, "On the complexity of computing the hypervolume indicator," IEEE Transactions on Evolutionary Computation, vol. 13, no. 5, pp. 1075-1082, 2009.

[31] M. Elarbi, S. Bechikh, A. Gupta, L. B. Said, and Y. Ong, "A new decomposition-based nsga-ii for many-objective optimization," IEEE Transactions on Systems, Man, and Cybernetics: Systems, vol. 48, no. 7, pp. 1191-1210, 2018.

[32] A. Trivedi, D. Srinivasan, K. Pal, C. Saha, and T. Reindl, "Enhanced multiobjective evolutionary algorithm based on decomposition for solving the unit commitment problem," IEEE Transactions on Industrial Informatics, vol. 11, no. 6, pp. 1346-1357, 2015.

[33] R. Akbari, R. Hedayatzadeh, K. Ziarati, and B. Hassanizadeh, "A multiobjective artificial bee colony algorithm," Swarm and Evolutionary Computation, vol. 2, pp. 39-52, 2012.

[34] W. Zhang and Y. Liu, "Multi-objective reactive power and voltage control based on fuzzy optimization strategy and fuzzy adaptive particle swarm," International Journal of Electrical Power \& Energy Systems, vol. 30, no. 9, pp. 525-532, 2008.

[35] Z. Chen and H. T. Zhang, "A minimal control multiagent for collision avoidance and velocity alignment," IEEE Transactions on Cybernetics, vol. 47, no. 8, pp. 2185-2192, 2017. 


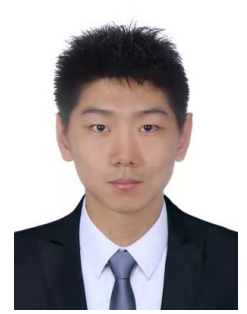

Runqi Chai (S'15-M'18) received the B.S. degree in information and computing science from the North China University of Technology, Beijing, China, in 2015 and the Ph.D. degree in Aerospace Engineering from Cranfield University, Cranfield, U.K, in August 2018. He is currently a research fellow at Cranfield University. His research interests include trajectory optimization, networked control systems, multi-agent control systems, and autonomous vehicle motion planning.

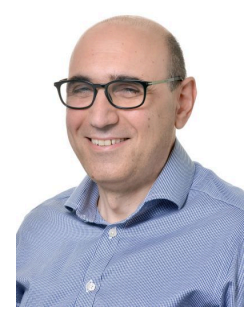

Antonios Tsourdos (M'99) obtained a Ph.D. on nonlinear robust missile autopilot design and analysis from Cranfield University, in 1999. He is a Professor of Autonomous Systems and Control with Cranfield University. He was appointed Head of the Autonomous Systems Group in 2007, Head of the Centre of Autonomous and Cyber-Physical Systems in 2012 and Director of Research - Aerospace, Transport and Manufacturing in 2015. He leads the research theme on autonomous systems within the School of Aerospace, Transport and Manufacturing at Cranfield University. He has diverse expertise in both unmanned and autonomous vehicles as well as networked systems.He conducts basic and applied research in the fields of guidance, control and navigation for single and multiple unmanned autonomous vehicles as well as research on cyberphysical systems.

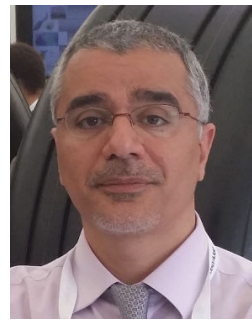

Al Savvaris received the M.Eng. degree in aerospace systems engineering from the University of Hertfordshire, Hertfordshire, U.K., in 1998 and the Ph.D. degree in radiowave propagation and system design from the University of South Wales, Pontypridd, U.K., in 2004. His research interests include trajectory optimization, networked control systems, and aircraft control systems.

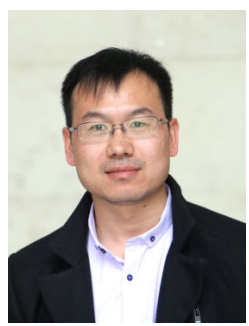

Senchun Chai (M'19-SM'19) received the B.S and Master degree from Beijing Institute of Technology, Beijing, China from 1997 to 2004 and the Ph.D. degree in Networked Control System from University of South Wales, Pontypridd, U.K., in 2007.

$\mathrm{He}$ is currently an associated professor of School of Automation with Beijing Institute of Technology His current research interests focus on flight control system, networked control systems, embedded systems and multi-agent control systems.

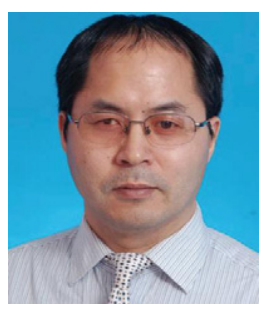

Yuanqing Xia (M'15-SM'16) was born in Anhui Province, China, in 1971. He received the B.S. degree from the Department of Mathematics, Chuzhou University, Chuzhou, China, in 1991, the M.S. degree in fundamental mathematics from Anhui University, Wuhu, China, in 1998, and the Ph.D. degree in control theory and control engineering from the Beijing University of Aeronautics and Astronautics, Beijing, China, in 2001. His current research interests are in the fields of networked control systems, robust control and signal processing, active disturbance rejection control and flight control. He has published 8 monographs with Springer and Wiley, and more than 200 papers in journals. He has obtained Second Award of the Beijing Municipal Science and Technology (No. 1) in 2010, Second National Award for Science and Technology (No. 2) in 2011, and Second Natural Science Award of The Ministry of Education (No. 1) in 2012. He is a Deputy Editor of the Journal of the Beijing Institute of Technology, Associate Editor of Acta Automatica Sinica, Control Theory and Applications, the International Journal of Innovative Computing, Information and Control, and the International Journal of Automation and Computing.

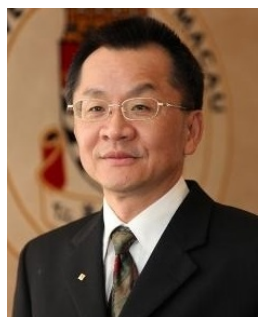

C. L. Philip Chen (S'88 - M'88 - SM'94 - F'07) received the M.S. degree in electrical engineering from the University of Michigan, Ann Arbor, MI, USA, in 1985 and the Ph.D. degree in electrical engineering from Purdue University, West Lafayette, IN, USA, in 1988.

$\mathrm{He}$ is a Chair Professor with the Department of Computer and Information Science, Faculty of Science and Technology, University of Macau, Macau, China. Being a Program Evaluator of the Accreditation Board of Engineering and Technology Education in the U.S., for computer engineering, electrical engineering, and software engineering programs, he successfully architects the University of Macau' $s$ Engineering and Computer Science programs receiving accreditations from Washington/Seoul Accord through Hong Kong Institute of Engineers (HKIE), of which is considered as his utmost contribution in engineering/computer science education for Macau as the former Dean of the Faculty. His current research interests include systems, cybernetics, and computational intelligence.

Dr. Chen was a recipient of the 2016 Outstanding Electrical and Computer Engineers Award from his alma mater, Purdue University, after he graduated from the University of Michigan at Ann Arbor, Ann Arbor, MI, USA. He was the IEEE SMC Society President from 2012 to 2013 and is currently a Vice President of Chinese Association of Automation (CAA). He is the Editor-inChief of the IEEE TRANSACTIONS ON SYSTEMS, MAN, AND CYBERNETICS: SYSTEMS and an Associate Editor of the IEEE TRANSACTIONS ON FUZZY SYSTEMS and IEEE TRANSACTIONS ON CYBERNETICS. He was the Chair of TC 9.1 Economic and Business Systems of International Federation of Automatic Control from 2015 to 2017. He is a fellow of AAAS, IAPR, CAA, and HKIE. 
2020-03-05

Multiobjective overtaking maneuver planning of autonomous ground vehicles

Chai, Runqi

IEEE

Chai R, Tsourdos A, Savvaris A, et al., (2021) Multiobjective overtaking maneuver planning of autonomous ground vehicles. IEEE Transactions on Cybernetics, IEEE Transactions on

Cybernetics, Volume 51, Issue 8, August 2021, pp. 4035-4049

https://doi.org/10.1109/TCYB.2020.2973748

Downloaded from Cranfield Library Services E-Repository 